The Free Internet Journal for Organic Chemistry
Paper

Arkivoc 2018, part iii, 120-133

Organic Chemistry

\title{
An efficient synthesis of
}

\section{3-diethoxyphosphoryl-4-(1H-indol-3-yl)-3,4-dihydrocoumarins: a convenient approach to 3-methylene-4-(indol-3-yl)-3,4-dihydrocoumarins}

\author{
Dariusz Deredas, * Henryk Krawczyk, and Krzysztof Huben
}

Institute of Organic Chemistry, Lodz University of Technology, Żeromskiego 116, 90-924 Łódź, Poland

Email: dariusz.deredas@p.lodz.pl

Received 12-01-2017

Accepted 12-29-2017

Published on line 01-28-2018

\section{Abstract}

TBD promoted conjugate addition of indoles to 3-diethoxyphosphorylcoumarins allows the synthesis 3diethoxyphosphoryl-4-(indol-3-yl)-3,4-dihydrocoumarins. The adducts derived from unsubstituted or C-5 methoxy substituted indole could be converted into the corresponding 3-methylene-(indol-3-yl)-3,4dihydrocoumarins by means of the HWE reaction with formaldehyde.

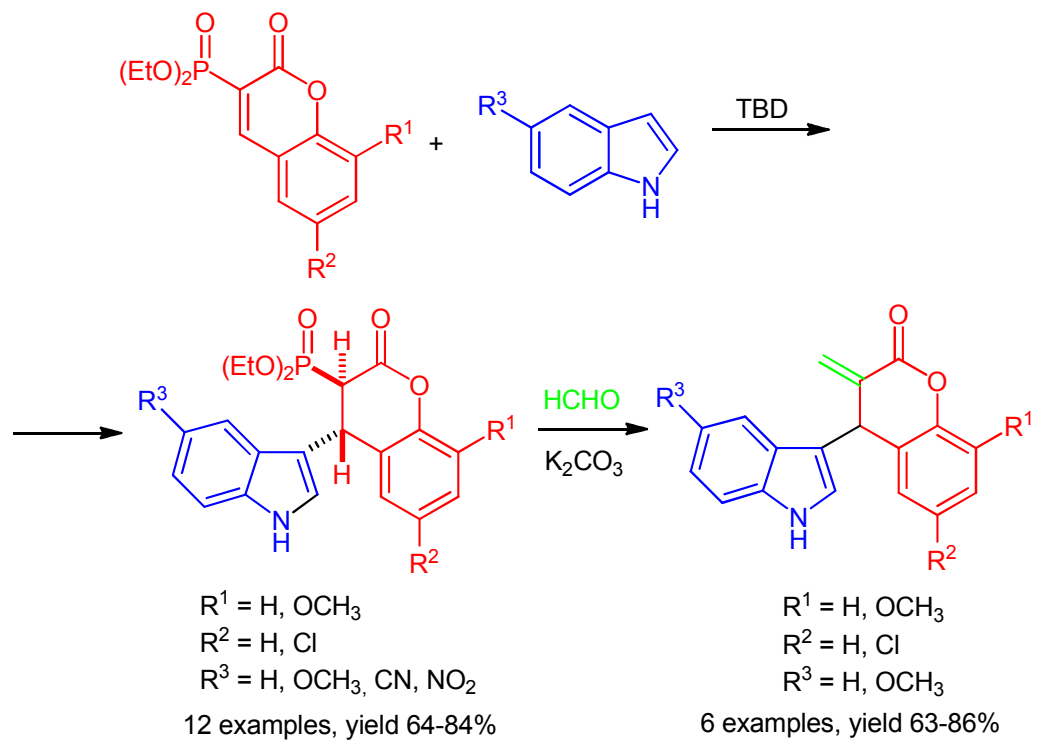

Keywords: Michael addition, HWE olefination, indoles, coumarins, 1,5,7-triazabicyclo[4.4.0]dec-5-ene (TBD) 


\section{Introduction}

Both indole and coumarin have been identified as privileged scaffolds in numerous biologically active molecules and natural products. ${ }^{1-3}$ Therefore, the development of synthetic methodologies enabling the synthesis of compounds containing both of these heterocyclic structures is highly desirable. A promising strategy to address this task is conjugate Michael-type addition of indoles to coumarins. Among several reactions of indole, the reactions involving C-3 functionalization of indole with electron-deficient olefins have attracted and continue to attract interest from the synthetic community. While the reaction of indoles with highly electrophilic nitroolefins and enones to furnish $\beta$-(indol-3-yl) alkylated products has been widely investigated, ${ }^{4-22}$ analogous reactions involving $\alpha, \beta$-unsaturated esters to form 3 -(indol-3-yl) alkanoates are rare. To date, two general strategies for the non-enantioselective synthesis of 3-(indol-3-yl) alkanoates have been reported. Ethyl 3-(indol-3-yl) alkanoates were obtained by one-pot, three-component KnoevenagelMichael reaction of indoles, Meldrum's acid and various aldehydes followed by decarboxylative ethanolysis of the adducts obtained. ${ }^{23-26}$ The other strategy is based on the conjugate addition of indoles to alkylidenemalonates. ${ }^{6}$ Recently, urea palladecycles ${ }^{27}$ and Sc(OTf) $)_{3} /$ sodium dodecyl sulfate ${ }^{28}$ have been demonstrated to be efficient Lewis acid catalysts for Friedel-Crafts alkylation of indoles with alkylidene malonates. Meanwhile, the catalytic asymmetric reactions of indoles with alkylidenemalonates have been reported. ${ }^{29-35}$ In sharp contrast, the conjugate addition of indoles to another class of doubly activated olefins, 3-EWG-coumarins, has rarely been reported. In fact, only two papers have been published, each containing a single entry, on the Lewis acid-catalysed conjugate addition of indoles to 3-ethoxycarbonylcoumarin. ${ }^{27,28}$ Moreover, in 2006 Tang et al. reported $\mathrm{Mg}(\mathrm{OTf})_{2}$-catalysed multicomponent tandem Michael additions of indoles with 3nitrocoumarins and methyl vinyl ketone leading to facile synthesis 3,3-disubstituted-4-(indol-3-yl)-3,4-dihydrocoumarins. ${ }^{36}$ Two efficient protocols for the synthesis of 3-unsubstituted-4-(indol-3-yl)-3,4-dihydrocoumarins have been reported. One of the methods utilises a one-pot three-component reaction of indoles, Meldrum's acid and salicylaldehyde. ${ }^{37}$ The other relies on cascade Michael addition/decarboxylation reactions of coumarin-3-carboxylic acids with indoles. ${ }^{38}$

\section{Results and Discussion}

We have recently discovered that conjugate addition of enolizable ketones to 3(diethoxyphosphoryl)coumarins is mediated by organic superbase 1,5,7-triazabicyclo[4.4.0]dec-5-ene (TBD). ${ }^{39,40}$ We envisioned that the use of the same approach would allow Michael addition of indole to 3(diethoxyphosphoryl)coumarins. Herein, this challenge has been addressed and we present the efficient synthesis of 3-diethoxyphosphoryl-4-(indol-3-yl)-3,4-dihydrocoumarins by an unprecedented TBD-mediated reaction of indoles with 3-(diethoxyphosphoryl)coumarins. We also demonstrate that in some cases the resulting adducts can be transformed into corresponding $\alpha$-methylene- $\delta$-lactones. Our initial attempts were focused on the synthesis of compound 3a. Preliminary experiments showed that TBD used in some excess is able to promote the smooth conjugate addition of indole $\mathbf{2 a}$ to coumarin $\mathbf{1 a}$. The reaction of coumarin $\mathbf{1} \mathbf{a}$ with 1.5 equivalents of indole $2 \mathrm{a}$ in the presence of two equivalents of TBD in $\mathrm{CH}_{2} \mathrm{Cl}_{2}$ at room temperature for 24 hours gave the best results in terms of yield and purity of the product 3a. After acidic quench, the crude product 3a was isolated as a mixture of two diastereoisomers in a ratio 1.0:0.1 (as indicated by ${ }^{31} \mathrm{P}-\mathrm{NMR}$ analysis) accompanied by unreacted coumarin (ca. 10\%). Notably the crystalline product $3 a$ was isolated as a sole trans-adduct after column chromatography in $84 \%$ yield. This indicates that diastereoisomeric products 
undergo rapid epimerization due to the presence of the acidic hydrogen at C-3. The protocol was successfully extended to a variety of coumarins and indoles (Scheme 1).<smiles>[R]c1cc([125I])c2oc(=O)c(P(=O)(OCC)OCC)cc2c1</smiles>

1a-c

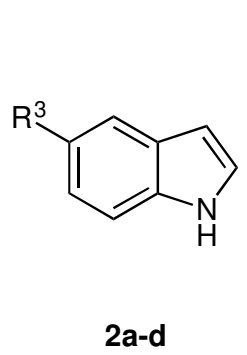

2a-d<smiles>[R]c1cc([R])c2c(c1)C(c1c[nH]c3ccc([R])cc13)C(=C)C(=O)O2</smiles>

3a-I 4a-f

Scheme 1. Reagents and conditions: (a) TBD (2.0 equiv), $\mathrm{CH}_{2} \mathrm{Cl}_{2}$, r.t., $24 \mathrm{~h}$; (b) $2 \mathrm{M}$ hydrochloric acid (excess), r.t.; (c) $\mathrm{K}_{2} \mathrm{CO}_{3}$ (3.0 equiv), THF, $0^{\circ} \mathrm{C}, 15$ min. then $\mathrm{CH}_{2} \mathrm{O}(40 \%$ aq), r.t., 3 h.

As summarized in Table 1 all reactions proceeded smoothly to give corresponding dihydrocoumarins 3a-I

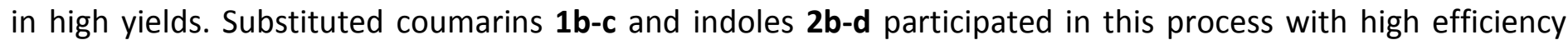
regardless of the presence of electron-withdrawing or electron-donating substituent on the aromatic ring. The crude products were formed as mixtures of trans- and cis-dihydrocoumarins. These mixtures were subjected to column chromatography to yield trans-adducts exclusively. The relative trans stereochemistry at the stereogenic centers C-3 and C-4 of 3-diethoxyphosphoryl-4-(indol-3-yl)-3,4-dihydrocoumarin 3a-I was assigned on the basis of ${ }^{13} \mathrm{C}-\mathrm{NMR}$ data. The observed values of the coupling constants ${ }^{3} \mathrm{~J}_{\mathrm{PC}(3)}=17.6-18.6 \mathrm{~Hz}$ clearly proved the trans arrangement of the phosphoryl and indolyl group. ${ }^{41,42}$ It is also worth noting that the formation of the corresponding $\mathrm{N}$-adducts was not observed under these reaction conditions.

Table 1. Yields of the compounds produced via Scheme 1

\begin{tabular}{cccccc}
\hline Entry & $\mathrm{R}^{1}$ & $\mathrm{R}^{2}$ & $\mathrm{R}^{3}$ & \multicolumn{2}{c}{ Yield (\%) } \\
\cline { 5 - 6 } & & & & $\mathbf{3}$ & $\mathbf{4}$ \\
\hline $\mathbf{a}$ & $\mathrm{H}$ & $\mathrm{H}$ & $\mathrm{H}$ & 84 & 64 \\
$\mathbf{b}$ & $\mathrm{OCH}_{3}$ & $\mathrm{H}$ & $\mathrm{H}$ & 64 & 68 \\
$\mathbf{c}$ & $\mathrm{H}$ & $\mathrm{Cl}$ & $\mathrm{H}$ & 67 & 63 \\
$\mathbf{d}$ & $\mathrm{H}$ & $\mathrm{H}$ & $\mathrm{OCH}_{3}$ & 77 & 86 \\
e & $\mathrm{OCH}_{3}$ & $\mathrm{H}$ & $\mathrm{OCH}_{3}$ & 74 & 70 \\
$\mathbf{f}$ & $\mathrm{H}$ & $\mathrm{Cl}$ & $\mathrm{OCH}_{3}$ & 83 & 71 \\
$\mathbf{g}$ & $\mathrm{H}$ & $\mathrm{H}$ & $\mathrm{NO}_{2}$ & 78 & - \\
$\mathbf{h}$ & $\mathrm{OCH}_{3}$ & $\mathrm{H}$ & $\mathrm{NO}_{2}$ & 69 & - \\
$\mathbf{i}$ & $\mathrm{H}$ & $\mathrm{Cl}$ & $\mathrm{NO}_{2}$ & 73 & - \\
$\mathbf{j}$ & $\mathrm{H}$ & $\mathrm{H}$ & $\mathrm{CN}$ & 81 & - \\
$\mathbf{k}$ & $\mathrm{OCH}_{3}$ & $\mathrm{H}$ & $\mathrm{CN}$ & 76 & - \\
$\mathbf{l}$ & $\mathrm{H}$ & $\mathrm{Cl}$ & $\mathrm{CN}$ & 80 & - \\
\hline
\end{tabular}


An examination of the HWE reaction of formaldehyde with phosphonolactones 3a-I revealed that the outcome of the reaction is determined by the electronic nature of the substituent present in the homoaromatic indole ring. The Horner-Wadsworth-Emmons (HWE) reaction of unsubstituted 3a-c and methoxy-substituted phosphonolactones 3d-f with formaldehyde in the presence of aqueous $\mathrm{K}_{2} \mathrm{CO}_{3}$ afforded the corresponding 3-methylene-4-(indol-3-yl)-3,4-dihydrocoumarins $\mathbf{4 a - c}$ and $\mathbf{4 d - f}$, respectively. On the other hand substrates $\mathbf{3 g}-\mathbf{I}$ and $\mathbf{3} \mathbf{j}-\mathbf{I}$ bearing an electron-withdrawing group at $\mathbf{C}-\mathbf{5}$ of the homoaromatic indole ring failed to give the desired methylenelactones $4 \mathrm{~g}-\mathrm{I}$ and only the products of the retro-Michael reaction were observed.<smiles>CCOC(=O)CP(=O)(OCC)OCC</smiles><smiles>CCOc1cccc2cc(P(=O)(O)OCC)c(=O)oc12</smiles>

8<smiles>C=C1C(=O)Oc2c(O[Ga-]C(C)C)cccc2C1c1c[nH]c2ccccc12</smiles>

10

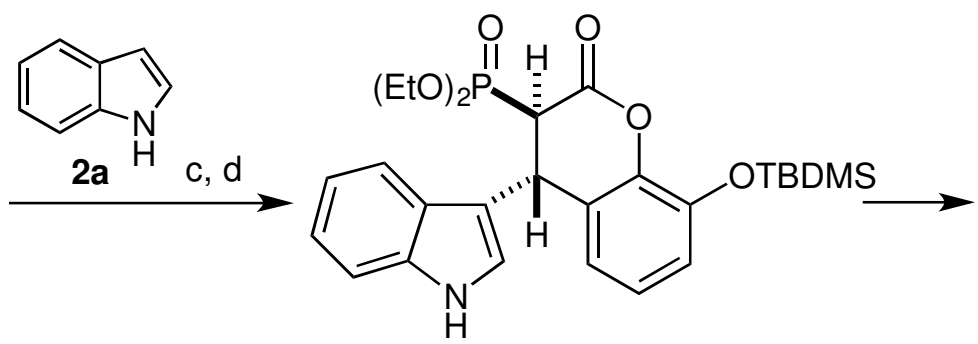

9<smiles>Cc1c(-c2c[nH]c3ccccc23)c2cccc(O)c2oc1=O</smiles>

11

Scheme 2. Reagents and conditions: (a) $\mathrm{CH}_{3} \mathrm{COOH}$ /piperidine (cat.), toluene, reflux, 15 h.; (b) TBDMSCl (1.1 equiv), imidazole (2.0 equiv), $\mathrm{CH}_{2} \mathrm{Cl}_{2}$, r.t., 24 h.; (c) TBD (2.0 equiv), $\mathrm{CH}_{2} \mathrm{Cl}_{2}$, r.t., 24 h; (d) $2 \mathrm{M}$ hydrochloric acid (excess), r.t.; (e) $\mathrm{K}_{2} \mathrm{CO}_{3}$ (3.0 equiv), THF, $0{ }^{\circ} \mathrm{C}, 15$ min. then $\mathrm{CH}_{2} \mathrm{O}(40 \%$ aq), r.t., 3 h.; (f) TBAF (1.1 equiv), THF, r.t., $3 \mathrm{~h}$.

Next, we extended the protocol of the Michael addition for 3-diethoxyphosphorylcoumarin 7 bearing an hydroxyl group in the aromatic ring (Scheme 2). Previously unknown 8-hydroxy-3-diethoxyphosphorylcoumarin 7 was readily prepared by Knoevenagel condensation of 2,3-dihydroxybenzaldehyde 5 with triethyl phosphonacetate 6 according to a classical procedure. ${ }^{43}$ We initially wanted to add indole to the unprotected coumarin 7, however attempted addition failed to give desired product. At that point the phenolic hydroxyl group in coumarin 7 was protected by silylation with $t$-BuMe ${ }_{2} \mathrm{SiCl}$. The silyl ether $\mathbf{8}$, stable both in basic and 
acidic conditions, was transformed cleanly into the desired protected trans 4-(indol-3-yl)-3,4-dihydrocoumarin 9 in 53\% yield. Finally, the HWE reaction of formaldehyde with 9 gave the corresponding protected 3methylene-4-(indol-3-yl)-3,4-dihydrocoumarin 10. Surprisingly, removal of the t-butyldimethylsilyl group by treatment with tetrabutylammonium fluoride in THF solution was accompanied by spontaneous exo-endo isomerization of the carbon-carbon double bond leading to 3-methyl-4-(indol-3-yl)coumarin 11. Recently, palladium-catalyzed coupling reactions of 4-cumarinyl triflates with indoles leading to the similar 4-(indol-3yl)coumarins have been reported. ${ }^{44}$

\section{Conclusions}

In summary, we have identified TBD as efficient promotor for Michael reaction of a variety of indoles with 3diethoxyphosphorylcoumarins. The HWE reaction of formaldehyde with the adducts bearing electron-rich indoles allowed facile preparation of methylene-4-(indol-3-yl)-3,4-dihydrocoumarins.

\section{Experimental Section}

General. NMR spectra were recorded on a Bruker Avance II Plus spectrometer at 700.0 MHz $\left({ }^{1} \mathrm{H}\right), 283.3 \mathrm{MHz}$ $\left({ }^{31} \mathrm{P}\right)$ and $176.0 \mathrm{MHz}\left({ }^{13} \mathrm{C}\right)$ respectively. Measurements were carried out in deuterochloroform (99.96\% $\mathrm{d}$, Aldrich) at $25^{\circ} \mathrm{C}$. Chemical shifts were calibrated relative to residual solvent peak $\left({ }^{1} \mathrm{H} \mathrm{NMR} \delta_{\mathrm{CHCl} 3}=7.26 \mathrm{ppm}\right.$ and $\left.{ }^{13} \mathrm{C} \mathrm{NMR} \delta_{\mathrm{CDCl} 3}=77.16 \mathrm{ppm}\right)$ and $85 \% \mathrm{H}_{3} \mathrm{PO}_{4}\left({ }^{31} \mathrm{P} \mathrm{NMR}\right)$. Chemical shifts are reported in ppm $(\delta), J$ values are given in $\mathrm{Hz}$. IR spectra were measured on Bruker Alpha FT-IR ATR spectrometer. Elemental analyses were performed on Perkin-Elmer PE 2400 analyser. Mass Spectrometry was carried out using Bruker amaZon speed EDT instrument. Melting points were determined in open capillaries on Büchi SMP 30 aparatus and were uncorrected. Flash chromatography was carried out using silica gel 60 (230-400 mesh). Thin layer chromatography was carried out on commercially available pre-coated plates (Fluka Silica gel on TLC plates).

General procedure for the synthesis of 3-diethoxyphosphoryl-4-(1H-indol-3-yl)-3,4-dihydrocoumarins (3a-I). To a stirred solution of the coumarin $1 \mathrm{a}-\mathrm{c}(1.0 \mathrm{mmol})$ and indole $2 \mathrm{a}-\mathrm{d}(1.5 \mathrm{mmol})$ in $\mathrm{CH}_{2} \mathrm{Cl}_{2}(10 \mathrm{~mL}), 1,5$,7triazabicyclo[4.4. 0]dec-5-ene $(0.278 \mathrm{~g}, 2.0 \mathrm{mmol})$ was added in one portion. Stirring was continued at $\mathrm{rt}$ for 24 $\mathrm{h}$. The resulting mixture was acidified with $5 \%$ hydrochloric acid $(10 \mathrm{~mL})$ and separated. The aqueous layer was extracted with $\mathrm{CH}_{2} \mathrm{Cl}_{2}(3 \times 20 \mathrm{~mL})$. The combined organic layers were washed with brine, dried over $\mathrm{MgSO}_{4}$ and evaporated. The oily residue was subjected for column chromatography on silica gel using $\mathrm{CH}_{2} \mathrm{Cl}_{2} / \mathrm{MeOH}(20: 1)$ as eluent $\left(R_{F} \sim 0.60-0.65\right)$ to give pure phosphonates 3a-I.

Diethyl $\left(\left(3 R^{*}, 4 S^{*}\right)-4-\left(1 H\right.\right.$-indol-3-yl)-2-oxochroman-3-yl)phosphonate (3a). Colorless crystals, mp $172-174{ }^{\circ} \mathrm{C}$; Anal. calcd for $\mathrm{C}_{21} \mathrm{H}_{22} \mathrm{NO}_{5} \mathrm{P}: \mathrm{C}, 63.15 ; \mathrm{H}, 5.55 ; \mathrm{N}, 3.51$; Found C, 63.2; H, 5.5; N, 3.5; IR(ATR): 3268, 2987, 1765, $1459,1242,1142,1061,1019,1006,964,943,738,516,428 \mathrm{~cm}^{-1} ; \delta_{\mathrm{H}}\left(700 \mathrm{MHz}_{\mathrm{CDCl}}\right) 8.21$ (bs, $\left.1 \mathrm{H}, \mathrm{NH}\right), 7.70$ $\left(\mathrm{d},{ }^{3} \mathrm{~J}_{\mathrm{HH}} 7.9 \mathrm{~Hz}, 1 \mathrm{H}, \mathrm{H}-\mathrm{C}_{\mathrm{Ar}}\right), 7.11-7.36\left(\mathrm{~m}, 7 \mathrm{H}, \mathrm{H}-\mathrm{C}_{\mathrm{Ar}}\right), 6.51\left(\mathrm{dd},{ }^{3} J_{\mathrm{HH}} 2.5 \mathrm{~Hz},{ }^{4} J_{\mathrm{HH}} 0.75 \mathrm{~Hz}, 1 \mathrm{H},-\mathrm{CH}-\mathrm{NH}-\right), 5.11(\mathrm{bd}$, $\left.{ }^{3} J_{\mathrm{PH}} 12.8 \mathrm{~Hz}, 1 \mathrm{H},-\mathrm{CH}-\mathrm{C}_{\mathrm{Ar}}\right), 4.11-4.21\left(\mathrm{~m}, 2 \mathrm{H},-\mathrm{CH}_{2}-\right), 3.88-3.92$ and 3.60-3.67 (m, 2H, $\left.-\mathrm{CH}_{2}-\right), 3.84\left(\mathrm{dd},{ }^{2} J_{\mathrm{PH}} 24.8\right.$ $\left.\mathrm{Hz},{ }^{3} J_{\mathrm{HH}} 1.1 \mathrm{~Hz}, 1 \mathrm{H}, \mathrm{H}-\mathrm{C}-\mathrm{P}\right), 1.33\left(\mathrm{dt},{ }^{3} J_{\mathrm{HH}} 7.1,{ }^{4} J_{\mathrm{PH}} 0.5 \mathrm{~Hz}, 3 \mathrm{H}, \mathrm{CH}_{3^{-}}\right), 0.98\left(\mathrm{dt},{ }^{3} J_{\mathrm{HH}} 7.1 \mathrm{~Hz},{ }^{4} J_{\mathrm{PH}} 0.5 \mathrm{~Hz}, 3 \mathrm{H}, \mathrm{CH}_{3^{-}}\right) ; \delta_{\mathrm{C}}$ $\left(176 \mathrm{MHz}, \mathrm{CDCl}_{3}\right) 163.8\left(\mathrm{~d},{ }^{2} J_{\mathrm{PC}} 5.7 \mathrm{~Hz}, \mathrm{O}-\mathrm{C}(\mathrm{O})\right), 151.7\left(C_{A r}\right), 136.8\left(C_{A r}\right), 129.1\left(C_{A r} \mathrm{H}\right), 129.0\left(C_{A r} \mathrm{H}\right), 125.2\left(C_{A r}\right)$, $125.2\left(C_{A r} \mathrm{H}\right), 123.3\left(C_{A r}\right), 122.9\left(C_{A r} \mathrm{H}\right), 122.1\left(C_{A r} \mathrm{H}\right), 120.3\left(C_{A r} \mathrm{H}\right), 118.3\left(C_{A r} \mathrm{H}\right), 116.9\left(C_{A r} \mathrm{H}\right), 116.8\left(d,{ }^{3} J_{P C} 18.0\right.$ $\left.\mathrm{Hz},-\mathrm{C}_{\mathrm{Ar}}-\mathrm{C}_{\mathrm{Ar}} \mathrm{H}-\mathrm{N}\right), 111.8\left(\mathrm{C}_{\mathrm{Ar}} \mathrm{H}\right), 63.5\left(\mathrm{~d},{ }^{2} \mathrm{~J}_{\mathrm{PC}} 6.5 \mathrm{~Hz}, \mathrm{CH}_{3}-\mathrm{CH}_{2}-\mathrm{OP}\right), 63.2\left(\mathrm{~d},{ }^{2} J_{\mathrm{PC}} 6.5 \mathrm{~Hz}, \mathrm{CH}_{3}-\mathrm{CH}_{2}-\mathrm{OP}\right), 47.5\left(\mathrm{~d},{ }^{1} J_{\mathrm{PC}}\right.$ 
123.5 Hz, $-\mathrm{CH}-\mathrm{P}$ ), 34.6 (d, ${ }^{3} \mathrm{~J}_{\mathrm{PC}} 3.0 \mathrm{~Hz},-\mathrm{C}_{\mathrm{Ar}}-\mathrm{CH}-\mathrm{C}_{\mathrm{Ar}}$ ), $16.4\left(\mathrm{~d},{ }^{3} \mathrm{~J}_{\mathrm{PC}} 6.2 \mathrm{~Hz}, \mathrm{CH}_{3}-\mathrm{CH}_{2}-\mathrm{OP}\right), 16.1\left(\mathrm{~d},{ }^{3} \mathrm{JPC}_{\mathrm{PC}} 6.2 \mathrm{~Hz} \mathrm{CH}_{3}-\mathrm{CH}_{2}-\right.$ OP); $\delta_{\mathrm{P}}\left(283.3 \mathrm{MHz}, \mathrm{CDCl}_{3}\right) 18.6 \mathrm{ppm}$.

Diethyl $\left(\left(3 R^{*}, 4 S^{*}\right)-4-(1 H\right.$-indol-3-yl)-8-methoxy-2-oxochroman-3-yl)phosphonate (3b). Colorless crystals, mp 160-162 ${ }^{\circ} \mathrm{C}$; Anal. calcd for $\mathrm{C}_{22} \mathrm{H}_{24} \mathrm{NO}_{6} \mathrm{P}: \mathrm{C}, 61.54 ; \mathrm{H}, 5.63 ; \mathrm{N}, 3.26$; Found $\mathrm{C}, 61.1 ; \mathrm{H}, 5.6 ; \mathrm{N}, 3.3 ; \mathrm{IR}(\mathrm{ATR}): 3272$, 2977, 1756, 1487, 1241, 1226, 1219, 1146, 1092, 1047, 966, 949, 737, $425 \mathrm{~cm}^{-1} ; \delta_{\mathrm{H}}\left(700 \mathrm{MHz}, \mathrm{CDCl}_{3}\right) 8.24$ (bs, $1 \mathrm{H}, \mathrm{NH}), 7.70\left(\mathrm{bd},{ }^{3} \mathrm{~J}_{\mathrm{HH}} 7.9 \mathrm{~Hz}, 1 \mathrm{H}, \mathrm{H}-\mathrm{C}_{\mathrm{Ar}}\right), 6.88-7.36\left(\mathrm{~m}, 6 \mathrm{H}, \mathrm{H}-\mathrm{C}_{\mathrm{Ar}}\right), 6.56\left(\mathrm{bd},{ }^{3} \mathrm{~J}_{\mathrm{HH}} 2.5 \mathrm{~Hz}, 1 \mathrm{H},-\mathrm{CH}-\mathrm{NH}-\right), 5.09(\mathrm{bd}$, $\left.{ }^{3} J_{\mathrm{PH}} 12.7 \mathrm{~Hz}, 1 \mathrm{H},-\mathrm{CH}-\mathrm{C}_{\mathrm{Ar}}\right), 4.11-4.20\left(\mathrm{~m}, 2 \mathrm{H},-\mathrm{CH}_{2}-\right), 3.86-3.93$ and 3.60-3.69 (m, 2H, $\left.-\mathrm{CH}_{2}-\right), 3.90\left(\mathrm{~s}, 3 \mathrm{H},-\mathrm{OCH}_{3}\right)$, $3.82\left(\mathrm{dd},{ }^{2} \mathrm{~J}_{\mathrm{PH}} 24.7 \mathrm{~Hz},{ }^{3} \mathrm{~J}_{\mathrm{HH}} 0.9 \mathrm{~Hz}, 1 \mathrm{H}, \mathrm{H}-\mathrm{C}-\mathrm{P}\right), 1.33\left(\mathrm{t},{ }^{3} \mathrm{~J}_{\mathrm{HH}} 7.1 \mathrm{~Hz}, 3 \mathrm{H}, \mathrm{CH}_{3^{-}}\right), 0.99\left(\mathrm{t},{ }^{3} \mathrm{~J}_{\mathrm{HH}} 7.1 \mathrm{~Hz}, 3 \mathrm{H}, \mathrm{CH}_{3^{-}}\right) ; \delta_{\mathrm{C}}(176$ $\left.\mathrm{MHz}, \mathrm{CDCl}_{3}\right) 163.2\left(\mathrm{~d},{ }^{2} J_{\mathrm{PC}} 5.7 \mathrm{~Hz}, \mathrm{O}-\mathrm{C}(\mathrm{O})\right), 147.6\left(C_{A r}\right), 141.1\left(C_{A r}\right), 136.8\left(C_{A r}\right), 125.3\left(C_{A r}\right), 125.1\left(C_{A r} \mathrm{H}\right), 124.4$ $\left(C_{A r}\right), 122.8\left(C_{A r} \mathrm{H}\right), 122.2\left(C_{A r} \mathrm{H}\right), 120.5\left(C_{A r} \mathrm{H}\right), 120.2\left(C_{A r} \mathrm{H}\right), 118.3\left(C_{A r} \mathrm{H}\right), 116.4\left(\mathrm{~d},{ }^{3} J_{\mathrm{PC}} 17.9 \mathrm{~Hz},-C_{A r}-C_{A r} \mathrm{H}-\mathrm{N}\right)$, $111.8\left(C_{A r} \mathrm{H}\right), 111.6\left(C_{A r} \mathrm{H}\right), 63.5\left(d,{ }^{2} J_{P C} 6.2 \mathrm{~Hz}, \mathrm{CH}_{3}-\mathrm{CH}_{2}-\mathrm{OP}\right), 63.2\left(\mathrm{~d},{ }^{2} J_{\mathrm{PC}}=6.2 \mathrm{~Hz}, \mathrm{CH}_{3}-\mathrm{CH}_{2}-\mathrm{OP}\right), 56.3(-\mathrm{OCH})_{3}$, $47.3\left(\mathrm{~d},{ }^{1} J_{\mathrm{PC}} 123.7 \mathrm{~Hz},-\mathrm{CH}-\mathrm{P}\right), 34.8$ (d, ${ }^{3} \mathrm{~J}_{\mathrm{PC}} 3.0 \mathrm{~Hz},-\mathrm{C}_{\mathrm{Ar}}-\mathrm{CH}-\mathrm{C}_{\mathrm{Ar}}$ ), 16.3 (d, ${ }^{3} \mathrm{~J}_{\mathrm{PC}} 6.2 \mathrm{~Hz}, \mathrm{CH}_{3}-\mathrm{CH}_{2}-\mathrm{OP}$ ), 16.0 (d, ${ }^{3} J_{\mathrm{PC}} 6.2$ $\left.\mathrm{Hz}, \mathrm{CH}_{3}-\mathrm{CH}_{2}-\mathrm{OP}\right) ; \delta_{\mathrm{P}}\left(283.3 \mathrm{MHz}, \mathrm{CDCl}_{3}\right) 19.4 \mathrm{ppm}$.

Diethyl $\left(\left(3 R^{*}, 4 S^{*}\right)-6\right.$-chloro-4-(1H-indol-3-yl)-2-oxochroman-3-yl)phosphonate (3c). Colorless crystals, mp 164-166 ${ }^{\circ} \mathrm{C}$; Anal. calcd for $\mathrm{C}_{21} \mathrm{H}_{21} \mathrm{CINO}_{5} \mathrm{P}: \mathrm{C}, 58.14 ; \mathrm{H}, 4.88 ; \mathrm{N}, 3.23$; found $\mathrm{C}, 58.1 ; \mathrm{H}, 4.9 ; \mathrm{N}, 3.2 ;$ IR(ATR): 3263, 2982, 2930, 1769, 1749, 1485, 1231, 1218, 1145, 1010, 979, 748, 739, $502 \mathrm{~cm}^{-1} ; \delta_{\mathrm{H}}\left(700 \mathrm{MHz}, \mathrm{CDCl}_{3}\right) 8.22$ (bs, $1 \mathrm{H}, \mathrm{NH}), 7.67\left(\mathrm{bd},{ }^{3} J_{\mathrm{HH}} 7.9 \mathrm{~Hz}, 1 \mathrm{H}, \mathrm{H}-\mathrm{C}_{\mathrm{Ar}}\right), 7.17-7.37\left(\mathrm{~m}, 6 \mathrm{H}, \mathrm{H}-\mathrm{C}_{\mathrm{Ar}}\right), 6.54\left(\mathrm{bd},{ }^{3} J_{\mathrm{HH}} 2.4 \mathrm{~Hz}, 1 \mathrm{H},-\mathrm{CH}-\mathrm{NH}-\right), 5.07(\mathrm{bd}$, $\left.{ }^{3} J_{\mathrm{PH}} 12.7 \mathrm{~Hz}, 1 \mathrm{H},-\mathrm{CH}-\mathrm{C}_{\mathrm{Ar}}\right), 4.13-4.22\left(\mathrm{~m}, 2 \mathrm{H},-\mathrm{CH}_{2}-\right), 3.92-3.99$ and 3.76-3.83 (m, 2H, $\left.-\mathrm{CH}_{2^{-}}\right), 3.81\left(\mathrm{dd},{ }^{2} \mathrm{~J}_{\mathrm{PH}} 24.7\right.$ $\left.\mathrm{Hz},{ }^{3} J_{\mathrm{HH}} 1.1 \mathrm{~Hz}, 1 \mathrm{H}, \mathrm{H}-\mathrm{C}-\mathrm{P}\right), 1.34\left(\mathrm{t},{ }^{3} \mathrm{~J}_{\mathrm{HH}} 7.0 \mathrm{~Hz}, 3 \mathrm{H}, \mathrm{CH}_{3^{-}}\right), 1.05\left(\mathrm{t},{ }^{3} \mathrm{~J}_{\mathrm{HH}} 7.0 \mathrm{~Hz}, 3 \mathrm{H}, \mathrm{CH}_{3^{-}}\right) ; \delta_{\mathrm{C}}\left(176 \mathrm{MHz} \mathrm{CDCl}_{3}\right)$ $163.2\left(\mathrm{~d},{ }^{2} J_{\mathrm{PC}} 5.7 \mathrm{~Hz}, \mathrm{O}-C(\mathrm{O})\right), 150.3\left(C_{A r}\right), 141.1\left(C_{A r}\right), 136.8\left(C_{A r}\right), 130.1\left(C_{A r}\right), 129.0\left(C_{A r} \mathrm{H}\right), 128.9\left(C_{A r} \mathrm{H}\right), 125.0(\mathrm{~d}$, ${ }^{2} J_{P C} 7.6 \mathrm{~Hz},\left(C_{A r}\right), 123.1\left(C_{A r} \mathrm{H}\right), 122.0\left(C_{A r} \mathrm{H}\right), 120.5\left(C_{A r} \mathrm{H}\right), 118.2\left(C_{A r} \mathrm{H}\right), 118.2\left(C_{A r} \mathrm{H}\right), 116.1\left(\mathrm{~d},{ }^{3} J_{P C} 17.8 \mathrm{~Hz},-C_{A r}\right.$ $\mathrm{C}_{\mathrm{Ar}} \mathrm{H}-\mathrm{N}$ ), $111.8\left(\mathrm{C}_{\mathrm{Ar}} \mathrm{H}\right), 63.7\left(\mathrm{~d},{ }^{2} \mathrm{~J}_{\mathrm{PC}} 6.4 \mathrm{~Hz}, \mathrm{CH}_{3}-\mathrm{CH}_{2}-\mathrm{OP}\right), 63.3\left(\mathrm{~d},{ }^{2} J_{\mathrm{PC}} 6.4 \mathrm{~Hz}, \mathrm{CH}_{3}-\mathrm{CH}_{2}-\mathrm{OP}\right), 47.2\left(\mathrm{~d},{ }^{1} J_{\mathrm{PC}} 123.7 \mathrm{~Hz},-\right.$ $\mathrm{CH}-\mathrm{P}$ ), 34.6 (d, ${ }^{3} J_{\mathrm{PC}} 3.0 \mathrm{~Hz},-\mathrm{C}_{\mathrm{Ar}}-\mathrm{CH}-\mathrm{C}_{\mathrm{Ar}}$ ), 16.3 (d, ${ }^{3} \mathrm{~J}_{\mathrm{PC}} 6.3 \mathrm{~Hz}, \mathrm{CH}_{3}-\mathrm{CH}_{2}-\mathrm{OP}$ ), 16.1 (d, ${ }^{3} J_{\mathrm{PC}} 6.3 \mathrm{~Hz}, \mathrm{CH}_{3}-\mathrm{CH}_{2}-\mathrm{OP}$ ); $\delta_{\mathrm{P}}$ (283.3 $\left.\mathrm{MHz} \mathrm{CDCl}_{3}\right) 19.1 \mathrm{ppm}$.

Diethyl $\left(\left(3 R^{*}, 4 S^{*}\right)\right.$-4-(5-methoxy-1H-indol-3-yl)-2-oxochroman-3-yl)phosphonate (3d). Colorless crystals, mp 158-160 ${ }^{\circ} \mathrm{C}$; Anal. calcd for $\mathrm{C}_{22} \mathrm{H}_{24} \mathrm{NO}_{6} \mathrm{P}: \mathrm{C}, 61.54 ; \mathrm{H}, 5.63 ; \mathrm{N}, 3.26$; Found $\mathrm{C}, 61.5 ; \mathrm{H}, 5.6 ; \mathrm{N}, 3.3 ; \mathrm{IR}(\mathrm{ATR}): 3304$, 2992, 2905, 1769, 1489, 1217, 1144, 1048, 1021, 1009, 930, 761, $512 \mathrm{~cm}^{-1} ; \delta_{\mathrm{H}}\left(700 \mathrm{MHz}, \mathrm{CDCl}_{3}\right) 7.98$ (bs, $1 \mathrm{H}$, $\mathrm{NH}), 7.10-7.38\left(\mathrm{~m}, 6 \mathrm{H}, \mathrm{H}-\mathrm{C}_{\mathrm{Ar}}\right), 6.89\left(\mathrm{dd},{ }^{3} \mathrm{~J}_{\mathrm{HH}} 8.7 \mathrm{~Hz},{ }^{4} J_{\mathrm{HH}} 2.3 \mathrm{~Hz}, 1 \mathrm{H}, \mathrm{H}-\mathrm{C}_{\mathrm{Ar}}\right), 6.51\left(\mathrm{bd},{ }^{3} J_{\mathrm{HH}} 1.9 \mathrm{~Hz}, 1 \mathrm{H},-\mathrm{CH}-\mathrm{NH}-\right)$, $5.06\left(\mathrm{bd},{ }^{3} J_{\mathrm{PH}} 12.8 \mathrm{~Hz}, 1 \mathrm{H},-\mathrm{CH}-\mathrm{C}_{\mathrm{Ar}}\right), 4.12-4.21\left(\mathrm{~m}, 2 \mathrm{H},-\mathrm{CH}_{2^{-}}\right), 3.85-3.92$ and 3.60-3.67 (m, $\left.2 \mathrm{H},-\mathrm{CH}_{2}-\right), 3.90(\mathrm{~s}, 3 \mathrm{H}$, $\left.-\mathrm{OCH}_{3}\right), 3.82\left(\mathrm{dd},{ }^{2} J_{\mathrm{PH}} 24.8 \mathrm{~Hz},{ }^{3} \mathrm{~J}_{\mathrm{HH}} 1.0 \mathrm{~Hz}, 1 \mathrm{H}, \mathrm{H}-\mathrm{C}-\mathrm{P}\right), 1.33\left(\mathrm{t},{ }^{3} J_{\mathrm{HH}} 7.0 \mathrm{~Hz}, 3 \mathrm{H}, \mathrm{CH}_{3^{-}}\right), 0.98\left(\mathrm{t},{ }^{3} \mathrm{JHH}_{\mathrm{HH}} 7.0 \mathrm{~Hz}, 3 \mathrm{H}, \mathrm{CH}_{3^{-}}\right.$ ); $\delta_{C}\left(176 \mathrm{MHz}, \mathrm{CDCl}_{3}\right) 163.8\left(\mathrm{~d},{ }^{2} J_{\mathrm{PC}} 5.2 \mathrm{~Hz}, \mathrm{O}-\mathrm{C}(\mathrm{O})\right), 154.7\left(C_{A r}\right), 151.7\left(C_{A r}\right), 131.9\left(C_{A r}\right), 129.1\left(C_{A r} \mathrm{H}\right), 129.0\left(C_{A r}\right)$, $125.7\left(C_{A r}\right), 125.2\left(C_{A r} \mathrm{H}\right), 123.3\left(C_{A r}\right), 122.7\left(C_{A r} \mathrm{H}\right), 116.9\left(C_{A r} \mathrm{H}\right), 116.7\left(\mathrm{~d},{ }^{3} J_{\mathrm{PC}} 18.3 \mathrm{~Hz},-C_{A r}-C_{A r} \mathrm{H}-\mathrm{N}\right), 113.2\left(C_{A r} \mathrm{H}\right)$, $112.5\left(C_{A r} \mathrm{H}\right), 100.2\left(C_{A r} \mathrm{H}\right), 63.5\left(\mathrm{~d},{ }^{2} J_{\mathrm{PC}} 6.2 \mathrm{~Hz}, \mathrm{CH}_{3}-\mathrm{CH}_{2}-\mathrm{OP}\right), 63.2\left(\mathrm{~d},{ }^{2} J_{\mathrm{PC}} 6.2 \mathrm{~Hz}, \mathrm{CH}_{3}-\mathrm{CH}_{2}-\mathrm{OP}\right), 56.1(-\mathrm{OCH})_{3}, 47.4$ (d, $\left.{ }^{1} J_{P C} 123.6 \mathrm{~Hz},-\mathrm{CH}-\mathrm{P}\right), 34.6\left(\mathrm{~d},{ }^{3} \mathrm{~J}_{\mathrm{PC}} 3.0 \mathrm{~Hz},-\mathrm{C}_{\mathrm{Ar}}-\mathrm{CH}-\mathrm{C}_{\mathrm{Ar}}\right), 16.4\left(\mathrm{~d},{ }^{3} \mathrm{~J}_{\mathrm{PC}} 6.2 \mathrm{~Hz}, \mathrm{CH}_{3}-\mathrm{CH}_{2}-\mathrm{OP}\right), 16.1\left(\mathrm{~d},{ }^{3} \mathrm{~J}_{\mathrm{PC}} 6.2 \mathrm{~Hz}\right.$, $\left.\mathrm{CH}_{3}-\mathrm{CH}_{2}-\mathrm{OP}\right) ; \delta_{\mathrm{P}}\left(283.3 \mathrm{MHz}, \mathrm{CDCl}_{3}\right) 19.6 \mathrm{ppm}$.

Diethyl $\left(\left(3 R,{ }^{*} 4 S^{*}\right)-8-m e t h o x y-4-(5-m e t h o x y-1 H\right.$-indol-3-yl)-2-oxochroman-3-yl)phosphonate (3e). Colorless crystals, mp 146-149 ${ }^{\circ} \mathrm{C}$; Anal. calcd for $\mathrm{C}_{23} \mathrm{H}_{26} \mathrm{NO}_{7} \mathrm{P}: \mathrm{C}, 60.13 ; \mathrm{H}, 5.70 ; \mathrm{N}, 3.05$; Found $\mathrm{C}, 60.1 ; \mathrm{H}, 5.7 ; \mathrm{N}, 3.1$; IR(ATR): 3256, 2982, 2936, 2903, 1755, 1484, 1210, 1147, 1091, 1046, 1009, 973, 794, 744, $525 \mathrm{~cm}^{-1} ; \delta_{H}(700$ $\left.\mathrm{MHz} \mathrm{CDCl}_{3}\right) 7.98$ (bs, $\left.1 \mathrm{H}, \mathrm{NH}\right), 6.60-7.25\left(\mathrm{~m}, 6 \mathrm{H}, \mathrm{H}-\mathrm{C}_{\mathrm{Ar}}\right), 6.55$ (bd, $\left.{ }^{3} J_{\mathrm{HH}} 2.5 \mathrm{~Hz}, 1 \mathrm{H},-\mathrm{CH}-\mathrm{NH}-\right), 5.04$ (bd, ${ }^{3} J_{\mathrm{PH}} 12.7$ $\left.\mathrm{Hz}, 1 \mathrm{H},-\mathrm{CH}-\mathrm{C}_{\mathrm{Ar}}\right), 4.12-4.21\left(\mathrm{~m}, 2 \mathrm{H},-\mathrm{CH}_{2^{-}}\right), 3.86-3.92$ and 3.62-3.69 (m, 2H, $\left.\left.-\mathrm{CH}_{2^{-}}\right), 3.91\left(\mathrm{~s}, 3 \mathrm{H},-\mathrm{OCH}_{3}\right),-\right), 3.88(\mathrm{~s}$, $3 \mathrm{H},-\mathrm{OCH}_{3}$ ), 3.80 (dd, $\left.{ }^{2} J_{\mathrm{PH}} 24.7 \mathrm{~Hz},{ }^{3} J_{\mathrm{HH}} 1.1 \mathrm{~Hz}, 1 \mathrm{H}, \mathrm{H}-\mathrm{C}-\mathrm{P}\right), 1.33\left(\mathrm{t},{ }^{3} J_{\mathrm{HH}} 7.0 \mathrm{~Hz}, 3 \mathrm{H}, \mathrm{CH}_{3^{-}}\right), 1.00\left(\mathrm{t},{ }^{3} J_{\mathrm{HH}} 7.0 \mathrm{~Hz}, 3 \mathrm{H}\right.$, $\left.\mathrm{CH}_{3^{-}}\right) ; \delta_{\mathrm{C}}\left(176 \mathrm{MHz}, \mathrm{CDCl}_{3}\right) 163.2\left(\mathrm{~d},{ }^{2}{ }_{\mathrm{PC}} 5.3 \mathrm{~Hz}, \mathrm{O}-\mathrm{C}(\mathrm{O})\right), 154.6\left(C_{A r}\right), 147.6\left(C_{A r}\right), 141.1\left(C_{A r}\right), 131.9\left(C_{A r}\right), 125.6$ $\left(C_{A r}\right), 125.1\left(C_{A r} \mathrm{H}\right), 124.4\left(C_{A r}\right), 122.8\left(C_{A r} \mathrm{H}\right), 120.5\left(C_{A r} \mathrm{H}\right), 116.1\left(\mathrm{~d},{ }^{3} J_{\mathrm{PC}} 17.9 \mathrm{~Hz},-C_{A r}-C_{A r} \mathrm{H}-\mathrm{N}\right), 113.0\left(C_{A r} \mathrm{H}\right)$, 112.5 $\left(C_{A r} \mathrm{H}\right), 111.6\left(C_{A r} \mathrm{H}\right), 100.1\left(C_{A r} \mathrm{H}\right), 63.4\left(\mathrm{~d},{ }^{2} J_{P C} 6.8 \mathrm{~Hz}, \mathrm{CH}_{3}-\mathrm{CH}_{2}-\mathrm{OP}\right), 63.2\left(\mathrm{~d},{ }^{2} J_{\mathrm{PC}} 6.8 \mathrm{~Hz}, \mathrm{CH}_{3}-\mathrm{CH}_{2}-\mathrm{OP}\right), 56.3$ 
$\left(-\mathrm{OCH}_{3}\right), 56.1\left(-\mathrm{OCH}_{3}\right), 47.2\left(\mathrm{~d},{ }^{1} J_{\mathrm{PC}} 123.4 \mathrm{~Hz},-\mathrm{CH}-\mathrm{P}\right), 34.8\left(\mathrm{~d},{ }^{3} \mathrm{~J}_{\mathrm{PC}} 2.6 \mathrm{~Hz},-\mathrm{C}_{\mathrm{Ar}}-\mathrm{CH}-\mathrm{C}_{\mathrm{Ar}}\right), 16.4\left(\mathrm{~d},{ }^{3} \mathrm{~J}_{\mathrm{PC}} 6.2 \mathrm{~Hz}, \mathrm{CH}_{3}-\right.$ $\mathrm{CH}_{2}-\mathrm{OP}$ ), 16.0 (d, $\left.{ }^{3} \mathrm{~J}_{\mathrm{PC}} 6.2 \mathrm{~Hz}, \mathrm{CH}_{3}-\mathrm{CH}_{2}-\mathrm{OP}\right) ; \delta_{\mathrm{P}}\left(283.3 \mathrm{MHz}, \mathrm{CDCl}_{3}\right) 18.5 \mathrm{ppm}$.

Diethyl ( $\left(3 R^{*}, 4 S^{*}\right)-6$-chloro-4-(5-methoxy-1H-indol-3-yl)-2-oxochroman-3-yl)phosphonate (3f). Colorless crystals, mp 192-194 ${ }^{\circ} \mathrm{C}$; Anal. calcd for $\mathrm{C}_{22} \mathrm{H}_{23} \mathrm{ClNO}_{6} \mathrm{P}: \mathrm{C}, 56.97 ; \mathrm{H}, 5.00 ; \mathrm{N}, 3.02$; Found C, 57.0; $\mathrm{H}, 5.0 ; \mathrm{N}, 3.0$; IR(ATR): 3393, 2992, 2903, 1768, 1485, 1228, 1218, 1144, 1048, 1015, 979, 820, 800, $507 \mathrm{~cm}^{-1} ; \delta_{\mathrm{H}}(700 \mathrm{MHz}$, $\left.\mathrm{CDCl}_{3}\right) 7.98(\mathrm{bs}, 1 \mathrm{H}, \mathrm{NH}), 6.86-7.40\left(\mathrm{~m}, 6 \mathrm{H}, \mathrm{H}-\mathrm{C}_{\mathrm{Ar}}\right), 6.53\left(\mathrm{bd},{ }^{3} \mathrm{~J}_{\mathrm{HH}} 2.5 \mathrm{~Hz}, 1 \mathrm{H},-\mathrm{CH}-\mathrm{NH}-\right), 5.01\left(\mathrm{bd},{ }^{3} \mathrm{~J}_{\mathrm{PH}} 12.9 \mathrm{~Hz}, 1 \mathrm{H}\right.$, $\left.-\mathrm{CH}-\mathrm{C}_{\mathrm{Ar}}\right), 4.13-4.22\left(\mathrm{~m}, 2 \mathrm{H},-\mathrm{CH}_{2}-\right), 3.92-3.99$ and 3.76-3.83 (m, 2H, $\left.-\mathrm{CH}_{2}-\right), 3.88\left(\mathrm{~s}, 3 \mathrm{H},-\mathrm{OCH}_{3}\right), 3.80\left(\mathrm{dd},{ }^{2} J_{\mathrm{PH}}\right.$ $\left.24.8 \mathrm{~Hz},{ }^{3} J_{\mathrm{HH}} 1.1 \mathrm{~Hz}, 1 \mathrm{H}, \mathrm{H}-\mathrm{C}-\mathrm{P}\right), 1.34\left(\mathrm{t},{ }^{3} J_{\mathrm{HH}} 7.0 \mathrm{~Hz}, 3 \mathrm{H}, \mathrm{CH}_{3^{-}}\right), 1.1\left(\mathrm{t},{ }^{3} J_{\mathrm{HH}} 7.0 \mathrm{~Hz}, 3 \mathrm{H}, \mathrm{CH}_{3^{-}}\right) ; \delta_{\mathrm{C}}\left(176 \mathrm{MHz} \mathrm{CDCl}_{3}\right)$ $163.2\left(\mathrm{~d},{ }^{2} J_{\mathrm{PC}} 5.0 \mathrm{~Hz}, \mathrm{O}-C(\mathrm{O})\right), 154.7\left(C_{A r}\right), 150.2\left(C_{A r}\right), 131.8\left(C_{A r}\right), 130.1\left(C_{A r}\right), 129.0\left(C_{A r} \mathrm{H}\right), 128.9\left(C_{A r} \mathrm{H}\right), 125.5$ $\left(C_{A r}\right), 125.0\left(C_{A r}\right), 122.6\left(C_{A r} \mathrm{H}\right), 118.2\left(C_{A r} \mathrm{H}\right), 115.9\left(\mathrm{~d},{ }^{3} J_{\mathrm{PC}} 17.6 \mathrm{~Hz},-C_{A r}-\mathrm{C}_{A r} \mathrm{H}-\mathrm{N}\right), 113.3\left(C_{A r} \mathrm{H}\right), 112.6\left(C_{A r} \mathrm{H}\right), 100.0$ $\left(C_{A r} \mathrm{H}\right), 63.7\left(\mathrm{~d},{ }^{2} J_{\mathrm{PC}} 6.7 \mathrm{~Hz}, \mathrm{CH}_{3}-\mathrm{CH}_{2}-\mathrm{OP}\right), 63.7\left(\mathrm{~d},{ }^{2} J_{\mathrm{PC}} 6.7 \mathrm{~Hz}, \mathrm{CH}_{3}-\mathrm{CH}_{2}-\mathrm{OP}\right), 56.1\left(-\mathrm{OCH}_{3}\right), 47.0\left(\mathrm{~d},{ }^{1} J_{\mathrm{PC}} 123.8 \mathrm{~Hz},-\right.$ $\mathrm{CH}-\mathrm{P}$ ), $34.6\left(\mathrm{~d},{ }^{3} \mathrm{~J}_{\mathrm{PC}}=2.6 \mathrm{~Hz},-\mathrm{C}_{\mathrm{Ar}}-\mathrm{CH}-\mathrm{C}_{\mathrm{Ar}}\right.$ ), $16.4\left(\mathrm{~d},{ }^{3} \mathrm{~J}_{\mathrm{PC}} 6.2 \mathrm{~Hz}, \mathrm{CH}_{3}-\mathrm{CH}_{2}-\mathrm{OP}\right), 16.1\left(\mathrm{~d},{ }^{3} J_{\mathrm{PC}} 6.2 \mathrm{~Hz} \mathrm{CH}_{3}-\mathrm{CH}_{2}-\mathrm{OP}\right) ; \delta_{\mathrm{P}}$ $\left(283.3 \mathrm{MHz}, \mathrm{CDCl}_{3}\right) 19.2 \mathrm{ppm}$.

Diethyl $\left(\left(3 R^{*}, 4 S^{*}\right)-4-(5-n i t r o-1 H\right.$-indol-3-yl)-2-oxochroman-3-yl)-phosphonate (3g). Colorless crystals, mp 185$188{ }^{\circ} \mathrm{C}$; Anal. calcd for $\mathrm{C}_{21} \mathrm{H}_{21} \mathrm{~N}_{2} \mathrm{O} 7 \mathrm{P}$ : C, 56.76; $\mathrm{H}, 4.76 ; \mathrm{N}, 6.30$; Found $\mathrm{C}, 56.8 ; \mathrm{H}, 4.7 ; \mathrm{N}, 6.3 ; \mathrm{IR}(\mathrm{ATR}): 3183$, 2984, 2927, 2907, 1753, 1517, 1479, 1335, 1227, 1152, 1009, 973, 767, 740, $506 \mathrm{~cm}^{-1} ; \delta_{\mathrm{H}}\left(700 \mathrm{MHz}^{\mathrm{CDCl}}{ }_{3}\right)$ 9.01 (bs, $1 \mathrm{H}, \mathrm{NH}), 8.62\left(\mathrm{~d},{ }^{4} J_{\mathrm{HH}} 2.0 \mathrm{~Hz}, 1 \mathrm{H}, \mathrm{C}-\mathrm{CH}-\mathrm{CNO}_{2}\right.$ ), 8.13 (dd, $\left.{ }^{3} J_{\mathrm{HH}} 8.9 \mathrm{~Hz},{ }^{4} J_{\mathrm{HH}} 2.0 \mathrm{~Hz}, 1 \mathrm{H}, \mathrm{HC}-\mathrm{CH}-\mathrm{CNO}_{2}\right), 7.40$ $\left(\mathrm{d},{ }^{3} J_{\mathrm{HH}} 8.9 \mathrm{~Hz}, 1 \mathrm{H}, \mathrm{CH}-\mathrm{CH}-\mathrm{CNO}_{2}\right), 7.11-7.38\left(\mathrm{~m}, 4 \mathrm{H}, \mathrm{H}-\mathrm{C}_{\mathrm{Ar}}\right), 6.71\left(\mathrm{bd},{ }^{3} J_{\mathrm{HH}} 2.3 \mathrm{~Hz}, 1 \mathrm{H},-\mathrm{CH}-\mathrm{NH}-\right)^{2}, 0.09\left(\mathrm{bd},{ }^{3} J_{\mathrm{PH}} 12.6\right.$ $\left.\mathrm{Hz}, 1 \mathrm{H},-\mathrm{CH}-\mathrm{C}_{\mathrm{Ar}}\right), 4.10-4.21\left(\mathrm{~m}, 2 \mathrm{H},-\mathrm{CH}_{2^{-}}\right), 3.92-3.99$ and 3.70-3.75 (m, 2H, $\left.-\mathrm{CH}_{2}-\right), 3.78\left(\mathrm{dd},{ }^{2} \mathrm{~J}_{\mathrm{PH}} 24.8 \mathrm{~Hz},{ }^{3} J_{\mathrm{HH}} 1.1\right.$ $\mathrm{Hz}, 1 \mathrm{H}, \mathrm{H}-\mathrm{C}-\mathrm{P}), 1.33\left(\mathrm{t},{ }^{3} \mathrm{~J}_{\mathrm{HH}} 7.1 \mathrm{~Hz}, 3 \mathrm{H}, \mathrm{CH}_{3^{-}}\right), 1.08\left(\mathrm{t},{ }^{3} J_{\mathrm{HH}} 7.1 \mathrm{~Hz}, 3 \mathrm{H}, \mathrm{CH}_{3^{-}}\right) ; \delta_{\mathrm{C}}\left(176 \mathrm{MHz}, \mathrm{CDCl}_{3}\right) 163.6\left(\mathrm{~d},{ }^{2} J_{\mathrm{PC}} 5.1\right.$ $\mathrm{Hz}$, O-C(O)), $151.6\left(C_{A r}\right), 142.2\left(C_{A r}\right), 139.8\left(C_{A r}\right), 129.5\left(C_{A r} \mathrm{H}\right), 129.0\left(C_{A r} \mathrm{H}\right), 125.5\left(C_{A r} \mathrm{H}\right), 125.4\left(C_{A r} \mathrm{H}\right), 124.7$ $\left(C_{A r}\right), 122.5\left(C_{A r}\right), 119.1\left(\mathrm{~d},{ }^{3} J_{\mathrm{PC}} 18.0 \mathrm{~Hz},-C_{A r}-\mathrm{C}_{\mathrm{Ar}} \mathrm{H}-\mathrm{N}\right), 118.5\left(C_{A r} \mathrm{H}\right), 117.1\left(C_{A r} \mathrm{H}\right), 115.5\left(C_{A r} \mathrm{H}\right), 112.0\left(C_{A r} \mathrm{H}\right), 63.7$ $\left(\mathrm{d},{ }^{2} J_{\mathrm{PC}} 6.6 \mathrm{~Hz}, \mathrm{CH}_{3}-\mathrm{CH}_{2}-\mathrm{OP}\right), 63.6\left(\mathrm{~d},{ }^{2} J_{\mathrm{PC}} 6.6 \mathrm{~Hz}, \mathrm{CH}_{3}-\mathrm{CH}_{2}-\mathrm{OP}\right), 48.8$ (d, $\left.{ }^{1} J_{\mathrm{PC}} 124.7 \mathrm{~Hz},-\mathrm{CH}-\mathrm{P}\right), 34.5$ (d, ${ }^{3} \mathrm{~J}_{\mathrm{PC}} 2.8 \mathrm{~Hz},-$ $\mathrm{C}_{\mathrm{Ar}}-\mathrm{CH}-\mathrm{C}_{\mathrm{Ar}}$ ), 16.4 (d, ${ }^{3} \mathrm{~J}_{\mathrm{PC}} 6.2 \mathrm{~Hz}, \mathrm{CH}_{3}-\mathrm{CH}_{2}-\mathrm{OP}$ ), 16.2 (d, ${ }^{3} \mathrm{JPC}_{\mathrm{PC}} 6.2 \mathrm{~Hz}, \mathrm{CH}_{3}-\mathrm{CH}_{2}-\mathrm{OP}$ ); $\delta_{\mathrm{P}}\left(283.3 \mathrm{MHz}, \mathrm{CDCl}_{3}\right) 18.7 \mathrm{ppm}$.

Diethyl $\quad\left(\left(3 R^{*}, 4 S^{*}\right)-8-m e t h o x y-4-(5-n i t r o-1 H\right.$-indol-3-yl)-2-oxochroman-3-yl)phosphonate (3h). Colorless crystals, mp $195-197{ }^{\circ} \mathrm{C}$; Anal. calcd for $\mathrm{C}_{22} \mathrm{H}_{23} \mathrm{~N}_{2} \mathrm{O}_{8} \mathrm{P}: \mathrm{C}, 55.70 ; \mathrm{H}, 4.89 ; \mathrm{N}, 5.91$; Found C, 55.8; $\mathrm{H}, 4.9 ; \mathrm{N}, 5.8$; IR(ATR): 3438, 2975, 2932, 2907, 1757, 1518, 1486, 1323, 1284, 1245, 1219, 1169, 1043, 1004, 971, 783, 738, $505 \mathrm{~cm}^{-1} ; \delta_{\mathrm{H}}\left(700 \mathrm{MHz}, \mathrm{CDCl}_{3}\right) 9.09$ (bs, $\left.1 \mathrm{H}, \mathrm{NH}\right), 8.61\left(\mathrm{~d},{ }^{4} J_{\mathrm{HH}} 2.1 \mathrm{~Hz}, 1 \mathrm{H}, \mathrm{C}-\mathrm{CH}-\mathrm{CNO}_{2}\right), 8.11\left(\mathrm{dd},{ }^{3} J_{\mathrm{HH}} 9.0 \mathrm{~Hz},{ }^{4} J_{\mathrm{HH}}\right.$ $\left.2.1 \mathrm{~Hz}, 1 \mathrm{H}, \mathrm{HC}-\mathrm{CH}-\mathrm{CNO}_{2}\right), 7.40\left(\mathrm{~d},{ }^{3} \mathrm{~J}_{\mathrm{HH}} 9.0 \mathrm{~Hz}, 1 \mathrm{H}, \mathrm{CH}-\mathrm{CH}-\mathrm{CNO}_{2}\right), 6.80-7.14\left(\mathrm{~m}, 3 \mathrm{H}, \mathrm{H}-\mathrm{C}_{\mathrm{Ar}}\right), 6.74\left(\mathrm{bd},{ }^{3} \mathrm{~J}_{\mathrm{HH}} 2.3 \mathrm{~Hz}\right.$, $1 \mathrm{H},-\mathrm{CH}-\mathrm{NH}-), 5.07\left(\mathrm{bd},{ }^{3} \mathrm{~J}_{\mathrm{PH}} 12.3 \mathrm{~Hz}, 1 \mathrm{H},-\mathrm{CH}-\mathrm{C}_{\mathrm{Ar}}\right), 4.12-4.21\left(\mathrm{~m}, 2 \mathrm{H},-\mathrm{CH}_{2^{-}}\right), 3.90-4.00$ and 3.71-3.78 (m, 2H, $\mathrm{CH}_{2^{-}}$), $3.87\left(\mathrm{~s}, 3 \mathrm{H},-\mathrm{OCH}_{3}\right), 3.77\left(\mathrm{dd},{ }^{2} \mathrm{~J}_{\mathrm{PH}} 24.8 \mathrm{~Hz},{ }^{3} \mathrm{~J}_{\mathrm{HH}} 1.1 \mathrm{~Hz}, 1 \mathrm{H}, \mathrm{H}-\mathrm{C}-\mathrm{P}\right), 1.34\left(\mathrm{t},{ }^{3} \mathrm{~J}_{\mathrm{HH}} 7.0 \mathrm{~Hz}, 3 \mathrm{H}, \mathrm{CH}_{3^{-}}\right), 1.09(\mathrm{t}$, $\left.{ }^{3} J_{\mathrm{HH}} 7.0 \mathrm{~Hz}, 3 \mathrm{H}, \mathrm{CH}_{3^{-}}\right) ; \delta_{\mathrm{C}}\left(176 \mathrm{MHz}, \mathrm{CDCl}_{3}\right) 163.3\left(\mathrm{~d},{ }^{2} J_{\mathrm{PC}} 4.9 \mathrm{~Hz}, 0-C(\mathrm{O})\right), 147.7\left(C_{A r}\right), 142.1\left(C_{A r}\right), 140.9\left(C_{A r}\right)$, $139.8\left(C_{A r}\right), 125.5\left(C_{A r} \mathrm{H}\right), 124.7\left(C_{A r}\right), 123.7\left(C_{A r}\right), 120.3\left(C_{A r} \mathrm{H}\right), 118.5\left(\mathrm{~d},{ }^{3} J_{\mathrm{PC}} 18.0 \mathrm{~Hz},-C_{A r}-C_{A r} \mathrm{H}-\mathrm{N}\right), 118.4\left(C_{A r} \mathrm{H}\right)$, $115.5\left(C_{A r} \mathrm{H}\right), 112.0\left(C_{A r} \mathrm{H}\right), 111.9\left(C_{A r} \mathrm{H}\right), 125.5125 .4\left(C_{A r} \mathrm{H}\right), 124.7\left(C_{A r}\right), 122.5\left(C_{A r}\right), 119.1118 .5\left(C_{A r} \mathrm{H}\right), 117.1$ $\left(C_{A r} \mathrm{H}\right), 115.5\left(C_{A r} \mathrm{H}\right), 112.0\left(C_{A r} \mathrm{H}\right), 63.7\left(d,{ }^{2} J_{P C} 6.6 \mathrm{~Hz}, \mathrm{CH}_{3}-\mathrm{CH}_{2}-\mathrm{OP}\right), 63.6\left(\mathrm{~d},{ }^{2} J_{\mathrm{PC}} 6.6 \mathrm{~Hz}, \mathrm{CH}_{3}-\mathrm{CH}_{2}-\mathrm{OP}\right), 56.2(-$ $\mathrm{OCH}_{3}$ ), $47.6\left(\mathrm{~d},{ }^{1} \mathrm{~J}_{\mathrm{PC}} 125.1 \mathrm{~Hz},-\mathrm{CH}-\mathrm{P}\right), 34.6\left(\mathrm{~d},{ }^{3} \mathrm{~J}_{\mathrm{PC}} 2.0 \mathrm{~Hz},-\mathrm{C}_{\mathrm{Ar}}-\mathrm{CH}-\mathrm{C}_{\mathrm{Ar}}\right), 16.3\left(\mathrm{~d},{ }^{3} \mathrm{~J}_{\mathrm{PC}} 6.1 \mathrm{~Hz}, \mathrm{CH}_{3}-\mathrm{CH}_{2}-\mathrm{OP}\right), 16.1(\mathrm{~d}$, $\left.{ }^{3} J_{\mathrm{PC}} 6.1 \mathrm{~Hz}, \mathrm{CH}_{3}-\mathrm{CH}_{2}-\mathrm{OP}\right) ; \delta_{\mathrm{P}}\left(283.3 \mathrm{MHz}, \mathrm{CDCl}_{3}\right) 18.7 \mathrm{ppm}$.

Diethyl ((3R* $\left.4 S^{*}\right)$-6-chloro-4-(5-nitro-1H-indol-3-yl)-2-oxochroman-3-yl)phosphonate (3i). Colorless crystals, mp 215-217 ${ }^{\circ} \mathrm{C}$; Anal. calcd for $\mathrm{C}_{21} \mathrm{H}_{20} \mathrm{ClN}_{2} \mathrm{O} 7 \mathrm{P}$ : C, 52.68; $\mathrm{H}, 4.21 ; \mathrm{N}, 7.40$; Found C, 52.8; H, 4.2; N, 7.4; IR(ATR): $3222,2982,1763,1476,1330,1243,1217,1149,1048,1025,739,509 \mathrm{~cm}^{-1} ; \delta_{\mathrm{H}}(700 \mathrm{MHz}$, acetone-d6) 10.90 (bs, $1 \mathrm{H}, \mathrm{NH}$ ), $8.67\left(\mathrm{~d},{ }^{4} J_{\mathrm{HH}} 2.1 \mathrm{~Hz}, 1 \mathrm{H}, \mathrm{C}-\mathrm{CH}-\mathrm{CNO}_{2}\right.$ ), 8.10 (dd, $\left.{ }^{3} J_{\mathrm{HH}} 9.0 \mathrm{~Hz},{ }^{4} J_{\mathrm{HH}} 2.1 \mathrm{~Hz}, 1 \mathrm{H}, \mathrm{HC}-\mathrm{CH}-\mathrm{CNO}_{2}\right), 7.65(\mathrm{~d}$, $\left.{ }^{4} J_{\mathrm{HH}} 2.5 \mathrm{~Hz}, 1 \mathrm{H}, \mathrm{C}-\mathrm{CH}-\mathrm{CCl}\right), 7.63\left(\mathrm{~d},{ }^{3} J_{\mathrm{HH}} 9.0 \mathrm{~Hz}, 1 \mathrm{H}, \mathrm{HC}-\mathrm{CH}-\mathrm{CNO}_{2}\right), 7.46\left(\mathrm{dd},{ }^{3} J_{\mathrm{HH}} 8.7 \mathrm{~Hz},{ }^{4} J_{\mathrm{HH}} 2.5 \mathrm{~Hz}, 1 \mathrm{H}, \mathrm{CH}-\mathrm{CH}-\right.$ $\mathrm{CCl}), 7.21\left(\mathrm{~d},{ }^{3} \mathrm{~J}_{\mathrm{HH}} 8.7 \mathrm{~Hz}, 1 \mathrm{H}, \mathrm{CH}-\mathrm{CH}-\mathrm{CCl}\right), 7.05$ (bs, $\left.1 \mathrm{H},-\mathrm{CH}-\mathrm{NH}-\right), 5.26$ (bd, $\left.{ }^{3} J_{\mathrm{PH}} 12.6 \mathrm{~Hz}, 1 \mathrm{H},-\mathrm{CH}-\mathrm{C}_{\mathrm{Ar}}\right), 4.13-4.20$ $\left(\mathrm{m}, 2 \mathrm{H},-\mathrm{CH}_{2^{-}}\right), 4.07-4.14$ and 3.83-3.90 (m, $\left.2 \mathrm{H},-\mathrm{CH}_{2^{-}}\right), 3.88\left(\mathrm{dd},{ }^{2} J_{\mathrm{PH}} 24.9 \mathrm{~Hz},{ }^{3} J_{\mathrm{HH}} 1.3 \mathrm{~Hz}, 1 \mathrm{H}, H-\mathrm{C}-\mathrm{P}\right), 1.30\left(\mathrm{t},{ }^{3} \mathrm{~J}_{\mathrm{HH}}\right.$ 
$\left.7.1 \mathrm{~Hz}, 3 \mathrm{H}, \mathrm{CH}_{3^{-}}\right), 1.01\left(\mathrm{t},{ }^{3} J_{\mathrm{HH}} 7.1 \mathrm{~Hz}, 3 \mathrm{H}, \mathrm{CH}_{3^{-}}\right)$; $\delta_{\mathrm{C}}\left(176 \mathrm{MHz}\right.$, acetone-d6) $163.2\left(\mathrm{~d},{ }^{2} J_{\mathrm{PC}} 5.2 \mathrm{~Hz}, 0-\mathrm{C}(\mathrm{O})\right), 151.5$ $\left(C_{A r}\right), 142.6\left(C_{A r}\right), 141.0\left(C_{A r}\right), 130.3\left(C_{A r}\right), 129.9\left(C_{A r} \mathrm{H}\right), 129.7\left(C_{A r} \mathrm{H}\right), 127.2\left(C_{A r} \mathrm{H}\right), 126.2\left(C_{A r}\right), 125.4\left(C_{A r}\right), 119.3$ $\left(C_{A r} \mathrm{H}\right), 119.2\left(\mathrm{~d},{ }^{3} J_{\mathrm{PC}} 18.0 \mathrm{~Hz},-C_{A r}-\mathrm{C}_{A r} \mathrm{H}-\mathrm{N}\right), 118.4\left(C_{A r} \mathrm{H}\right), 116.2\left(C_{A r} \mathrm{H}\right), 113.2\left(C_{A r} \mathrm{H}\right), 64.0\left(\mathrm{~d},{ }^{2} \mathrm{JPC}_{\mathrm{PC}} 6.5 \mathrm{~Hz}, \mathrm{CH}_{3}-\mathrm{CH}_{2-}\right.$ OP), 63.7 (d, $\left.{ }^{2} J_{P C} 6.5 \mathrm{~Hz}, \mathrm{CH}_{3}-\mathrm{CH}_{2}-\mathrm{OP}\right), 48.2\left(\mathrm{~d},{ }^{1} J_{\mathrm{PC}} 123.3 \mathrm{~Hz},-\mathrm{CH}-\mathrm{P}\right), 35.0\left(\mathrm{~d},{ }^{3} J_{\mathrm{PC}} 3.4 \mathrm{~Hz},-\mathrm{C}_{\mathrm{Ar}}-\mathrm{CH}-\mathrm{C}_{\mathrm{Ar}}\right), 16.5\left(\mathrm{~d},{ }^{3} J_{\mathrm{PC}}\right.$ $6.2 \mathrm{~Hz}, \mathrm{CH}_{3}-\mathrm{CH}_{2}-\mathrm{OP}$ ), 16.4 (d, ${ }^{3} \mathrm{~J}_{\mathrm{PC}} 6.2 \mathrm{~Hz}, \mathrm{CH}_{3}-\mathrm{CH}_{2}-\mathrm{OP}$ ); $\delta_{\mathrm{P}}(283.3 \mathrm{MHz}$, acetone-d6) $17.1 \mathrm{ppm}$.

Diethyl ((3R* $\left.4 S^{*}\right)-4-(5-c y a n o-1 H$-indol-3-yl)-2-oxochroman-3-yl)phosphonate (3j). Colorless crystals, mp 223$224{ }^{\circ} \mathrm{C}$; Anal. calcd for $\mathrm{C}_{22} \mathrm{H}_{21} \mathrm{~N}_{2} \mathrm{O}_{5} \mathrm{P}$ : C, 62.26; $\mathrm{H}, 4.99 ; \mathrm{N}, 6.60$; Found $\mathrm{C}, 62.3 ; \mathrm{H}, 5.1 ; \mathrm{N}, 6.6 ; \mathrm{IR}(\mathrm{ATR}): 3226$, 2990, 2904, 2222, 1754, 1228, 1167, 1022, 1011, 806, 771, $505 \mathrm{~cm}^{-1} ; \delta_{\mathrm{H}}\left(700 \mathrm{MHz}_{\mathrm{CDCl}}\right) 8.83$ (bs, $\left.1 \mathrm{H}, \mathrm{NH}\right)$, 8.00-8.01 (m, $1 \mathrm{H}, \mathrm{C}-\mathrm{CH}-\mathrm{CCN}), 7.45$ (dd, ${ }^{3} \mathrm{~J}_{\mathrm{HH}} 8.5 \mathrm{~Hz},{ }^{4} J_{\mathrm{HH}} 1.5 \mathrm{~Hz}, 1 \mathrm{H}, \mathrm{HC}-\mathrm{CH}-\mathrm{CCN}$ ), 7.41 (dd, ${ }^{3} J_{\mathrm{HH}} 8.5 \mathrm{~Hz},{ }^{5} J_{\mathrm{HH}} 0.6 \mathrm{~Hz}$, $1 \mathrm{H}, \mathrm{CH}-\mathrm{CH}-\mathrm{CCN}), 7.10-7.36\left(\mathrm{~m}, 4 \mathrm{H}, \mathrm{H}-\mathrm{C}_{\mathrm{Ar}}\right), 6.71\left(\mathrm{dd},{ }^{3}{ }_{\mathrm{HH}} 2.6 \mathrm{~Hz},{ }^{4} J_{\mathrm{HH}} 0.9 \mathrm{~Hz}, 1 \mathrm{H},-\mathrm{CH}-\mathrm{NH}-\right), 5.07\left(\mathrm{bd},{ }^{3} \mathrm{~J}_{\mathrm{PH}} 12.6 \mathrm{~Hz}\right.$, $\left.1 \mathrm{H},-\mathrm{CH}-\mathrm{C}_{\mathrm{Ar}}\right), 4.10-4.22\left(\mathrm{~m}, 2 \mathrm{H},-\mathrm{CH}_{2}-\right), 3.87-3.92$ and 3.63-3.70 (m, $\left.2 \mathrm{H},-\mathrm{CH}_{2}-\right), 3.73\left(\mathrm{dd},{ }^{2} J_{\mathrm{PH}} 24.8 \mathrm{~Hz},{ }^{3} J_{\mathrm{HH}} 1.1 \mathrm{~Hz}\right.$, $1 \mathrm{H}, \mathrm{H}-\mathrm{C}-\mathrm{P}), 1.33\left(\mathrm{t},{ }^{3} \mathrm{~J}_{\mathrm{HH}} 7.1 \mathrm{~Hz}, 3 \mathrm{H}, \mathrm{CH}_{3^{-}}\right), 1.02\left(\mathrm{t},{ }^{3} \mathrm{~J}_{\mathrm{HH}} 7.1 \mathrm{~Hz}, 3 \mathrm{H}, \mathrm{CH}_{3^{-}}\right) ; \delta_{\mathrm{C}}\left(176 \mathrm{MHz} \mathrm{CDCl}_{3}\right) 163.6\left(\mathrm{~d},{ }^{2} J_{\mathrm{PC}} 5.1 \mathrm{~Hz}\right.$, O-C(O)), $151.6\left(C_{A r}\right), 138.5\left(C_{A r}\right), 129.4\left(C_{A r} \mathrm{H}\right), 129.0\left(C_{A r} \mathrm{H}\right), 125.8\left(C_{A r} \mathrm{H}\right), 125.5\left(C_{A r} \mathrm{H}\right), 125.1\left(C_{A r}\right), 124.5\left(C_{A r} \mathrm{H}\right)$, $123.9\left(C_{A r} \mathrm{H}\right), 122.5\left(C_{A r}\right), 120.5\left(C_{A r}\right), 117.6\left(\mathrm{~d},{ }^{3} J_{\mathrm{PC}} 18.3 \mathrm{~Hz},-C_{A r}-C_{A r} \mathrm{H}-\mathrm{N}\right), 117.1\left(C_{A r} \mathrm{H}\right), 112.8\left(C_{A r} \mathrm{H}\right), 103.5\left(C_{A r}\right)$, $63.7\left(d,{ }^{2} J_{P C} 6.6 \mathrm{~Hz}, \mathrm{CH}_{3}-\mathrm{CH}_{2}-\mathrm{OP}\right.$ ), 63.5 (d, ${ }^{2} J_{\mathrm{PC}} 6.6 \mathrm{~Hz}, \mathrm{CH}_{3}-\mathrm{CH}_{2}-\mathrm{OP}$ ), 48.6 (d, ${ }^{1} J_{\mathrm{PC}} 124.7 \mathrm{~Hz},-\mathrm{CH}-\mathrm{P}$ ), 34.4 (d, ${ }^{3} J_{P C} 2.1$ $\mathrm{Hz},-\mathrm{C}_{\mathrm{Ar}}-\mathrm{CH}-\mathrm{C}_{\mathrm{Ar}}$ ), 16.4 (d, $\left.{ }^{3} J_{\mathrm{PC}} 6.2 \mathrm{~Hz}, \mathrm{CH}_{3}-\mathrm{CH}_{2}-\mathrm{OP}\right), 16.1$ (d, ${ }^{3} J_{\mathrm{PC}} 6.2 \mathrm{~Hz}, \mathrm{CH}_{3}-\mathrm{CH}_{2}-\mathrm{OP}$ ); $\delta_{\mathrm{P}}\left(283.3 \mathrm{MHz}, \mathrm{CDCl}_{3}\right) 18.9$ ppm.

Diethyl ( $\left(3 R^{*}, 4 S^{*}\right)-4-(5-c y a n o-1 H$-indol-3-yl)-8-methoxy-2-oxochroman-3-yl)phosphonate (3k). Colorless crystals, mp 205-207 ${ }^{\circ} \mathrm{C}$; Anal. calcd for $\mathrm{C}_{23} \mathrm{H}_{23} \mathrm{~N}_{2} \mathrm{O}_{6} \mathrm{P}: \mathrm{C}, 60.79 ; \mathrm{H}, 5.10 ; \mathrm{N}, 6.16$; Found C, 60.1; $\mathrm{H}, 5.1 ; \mathrm{N}, 6.1$; IR(ATR): 3237, 2991, 2942, 2903, 2842, 2223, 1753, 1424, 1222, 1175, 1149, 1013, 806, 769, $494 \mathrm{~cm}^{-1} ; \delta_{\mathrm{H}}(700$ $\left.\mathrm{MHz} \mathrm{CDCl}_{3}\right) 8.87$ (bs, $\left.1 \mathrm{H}, \mathrm{NH}\right), 7.99-8.01(\mathrm{~m}, 1 \mathrm{H}, \mathrm{C}-\mathrm{CH}-\mathrm{CCN}), 7.44$ (dd, $\left.{ }^{3} \mathrm{~J}_{\mathrm{HH}} 8.4 \mathrm{~Hz},{ }^{4} J_{\mathrm{HH}} 1.5 \mathrm{~Hz}, 1 \mathrm{H}, \mathrm{HC}-\mathrm{CH}-\mathrm{CCN}\right)$, $7.41\left(\mathrm{dd},{ }^{3} \mathrm{~J}_{\mathrm{HH}} 8.4 \mathrm{~Hz},{ }^{5} \mathrm{JHH}_{\mathrm{HH}} 0.7 \mathrm{~Hz}, 1 \mathrm{H}, \mathrm{CH}-\mathrm{CH}-\mathrm{CCN}\right), 7.11$ (dd, $\left.{ }^{3} \mathrm{~J}_{\mathrm{HH}}{ }^{3} \mathrm{~J}_{\mathrm{HH}}=8.0 \mathrm{~Hz},-\mathrm{CH}-\mathrm{CH}-\mathrm{CH}-\right), 6.91\left(\mathrm{~d},{ }^{3} \mathrm{~J}_{\mathrm{HH}} 8.0,2 \mathrm{H}\right.$, $2 \mathrm{x}-\mathrm{CH}-\mathrm{CH}), 6.69\left(\mathrm{bd},{ }^{3} \mathrm{~J}_{\mathrm{HH}} 2.6 \mathrm{~Hz},{ }^{5} \mathrm{~J}_{\mathrm{HH}} 0.8 \mathrm{~Hz}, 1 \mathrm{H},-\mathrm{CH}-\mathrm{NH}-\right), 5.04\left(\mathrm{bd},{ }^{3} \mathrm{~J}_{\mathrm{PH}} 12.3 \mathrm{~Hz}, 1 \mathrm{H},-\mathrm{CH}-\mathrm{C}_{\mathrm{Ar}}\right), 4.12-4.21(\mathrm{~m}, 2 \mathrm{H}$, $-\mathrm{CH}_{2}$ ) , 3.89-3.95 and 3.65-3.71 (m, 2H, $\left.-\mathrm{CH}_{2^{-}}\right), 3.88\left(\mathrm{~s}, 3 \mathrm{H},-\mathrm{OCH}_{3}\right), 3.72\left(\mathrm{dd},{ }^{2} \mathrm{~J}_{\mathrm{PH}} 24.7 \mathrm{~Hz},{ }^{3} \mathrm{~J}_{\mathrm{HH}} 1.2 \mathrm{~Hz}, 1 \mathrm{H}, \mathrm{H}-\mathrm{C}-\mathrm{P}\right)$, $1.34\left(\mathrm{t},{ }^{3} J_{\mathrm{HH}} 7.0 \mathrm{~Hz}, 3 \mathrm{H}, \mathrm{CH}_{3^{-}}\right), 1.03\left(\mathrm{t},{ }^{3} J_{\mathrm{HH}} 7.0 \mathrm{~Hz}, 3 \mathrm{H}, \mathrm{CH}_{3^{-}}\right) ; \delta_{\mathrm{C}}\left(176 \mathrm{MHz}, \mathrm{CDCl}_{3}\right) 163.2\left(\mathrm{~d},{ }^{2} J_{\mathrm{PC}} 5.1 \mathrm{~Hz}, \mathrm{O}-\mathrm{C}(\mathrm{O})\right)$, 147.7 $\left(C_{A r}\right), 140.9\left(C_{A r}\right), 141.1\left(C_{A r}\right), 138.5\left(C_{A r}\right), 125.7\left(C_{A r} \mathrm{H}\right), 125.4\left(C_{A r} \mathrm{H}\right), 125.1\left(C_{A r}\right), 124.6\left(C_{A r} \mathrm{H}\right), 123.8\left(C_{A r} \mathrm{H}\right)$, $123.7\left(C_{A r}\right), 120.5\left(C_{A r}\right), 120.3\left(C_{A r} \mathrm{H}\right), 117.1\left(\mathrm{~d},{ }^{3} J_{\mathrm{PC}} 18.6 \mathrm{~Hz},-C_{A r}-C_{A r} \mathrm{H}-\mathrm{N}\right), 112.9\left(C_{A r} \mathrm{H}\right), 111.9\left(C_{A r} \mathrm{H}\right), 103.4\left(C_{A r}\right)$, $63.7\left(d,{ }^{2} J_{P C} 6.6 \mathrm{~Hz}, \mathrm{CH}_{3}-\mathrm{CH}_{2}-\mathrm{OP}\right), 63.5\left(\mathrm{~d},{ }^{2} J_{\mathrm{PC}} 6.6 \mathrm{~Hz}, \mathrm{CH}_{3}-\mathrm{CH}_{2}-\mathrm{OP}\right), 56.2\left(-\mathrm{OCH}_{3}\right), 47.5$ (d, $\left.{ }^{1} J_{P C} 125.1 \mathrm{~Hz},-\mathrm{CH}-\mathrm{P}\right)$, $34.5\left(\mathrm{~d},{ }^{3} J_{\mathrm{PC}} 2.1 \mathrm{~Hz},-\mathrm{C}_{\mathrm{Ar}}-\mathrm{CH}-\mathrm{C}_{\mathrm{Ar}}\right.$ ), 16.4 (d, $\left.{ }^{3} \mathrm{~J}_{\mathrm{PC}} 6.2 \mathrm{~Hz}, \mathrm{CH}_{3}-\mathrm{CH}_{2}-\mathrm{OP}\right), 16.0$ (d, $\left.{ }^{3} \mathrm{~J}_{\mathrm{PC}} 6.2 \mathrm{~Hz}, \mathrm{CH}_{3}-\mathrm{CH}_{2}-\mathrm{OP}\right) ; \delta_{P}(283.3$ $\left.\mathrm{MHz}, \mathrm{CDCl}_{3}\right) 17.7 \mathrm{ppm}$.

Diethyl $\left(\left(3 R^{*}, 4 S^{*}\right)-6\right.$-chloro-4-(5-cyano-1H-indol-3-yl)-2-oxochroman-3-yl)phosphonate (3I). Colorless crystals, mp 208-210 ${ }^{\circ} \mathrm{C}$; Anal. calcd for $\mathrm{C}_{22} \mathrm{H}_{20} \mathrm{ClN}_{2} \mathrm{O}_{5} \mathrm{P}: \mathrm{C}, 57.59 ; \mathrm{H}, 4.39 ; \mathrm{N}, 7.73$; Found C, 57.7; H, 4.4; N, 7.7; IR(ATR): 3336, 2983, 2940, 2908, 2223, 1765, 1479, 1419, 1253, 1226, 1138, 1053, 1014, 975, 811, 651, 513, $492 \mathrm{~cm}^{-1}$; $\delta_{\mathrm{H}}(700 \mathrm{MHz}$, acetone-d6) 10.78 (bs, $1 \mathrm{H}, \mathrm{NH}), 8.14$ (bs, $\left.1 \mathrm{H}, \mathrm{C}-\mathrm{CH}-\mathrm{CCN}\right), 7.65$ (d, $\left.{ }^{4} \mathrm{JHH}_{\mathrm{HH}} 2.5 \mathrm{~Hz}, 1 \mathrm{H},-\mathrm{C}-\mathrm{CH}-\mathrm{CCl}\right), 7.62$ (d, ${ }^{3} J_{\mathrm{HH}} 8.4 \mathrm{~Hz}, 1 \mathrm{H}, \mathrm{CH}-\mathrm{CH}-\mathrm{CCN}$ ), 7.50 (dd, ${ }^{3} J_{\mathrm{HH}} 8.4 \mathrm{~Hz},{ }^{5} J_{\mathrm{HH}} 1.3 \mathrm{~Hz} 1 \mathrm{H}, \mathrm{CH}-\mathrm{CH}-\mathrm{CCN}$ ), 7.44 (dd, ${ }^{3} J_{\mathrm{HH}} 8.8 \mathrm{~Hz},{ }^{5} J_{\mathrm{HH}} 2.5$ $\mathrm{Hz} 1 \mathrm{H},-\mathrm{C}-\mathrm{CH}-\mathrm{CH}-\mathrm{CCl}), 7.20$ (d, $\left.{ }^{3} \mathrm{~J}_{\mathrm{HH}} 8.8 \mathrm{~Hz}, 1 \mathrm{H},-\mathrm{C}-\mathrm{CH}-\mathrm{CH}-\mathrm{CCl}\right), 6.99$ (bs, $\left.1 \mathrm{H},-\mathrm{CH}-\mathrm{NH}-\right), 5.18\left(\mathrm{bd},{ }^{3} \mathrm{~J}_{\mathrm{PH}} 12.6 \mathrm{~Hz}, 1 \mathrm{H},-\right.$ $\left.\mathrm{CH}-\mathrm{C}_{\mathrm{Ar}}\right), 4.13-4.24\left(\mathrm{~m}, 2 \mathrm{H},-\mathrm{CH}_{2^{-}}\right), 3.94-4.03$ and 3.80-3.86 (m, $\left.2 \mathrm{H},-\mathrm{CH}_{2^{-}}\right), 3.89\left(\mathrm{dd},{ }^{2} J_{\mathrm{PH}} 24.9 \mathrm{~Hz},{ }^{3} \mathrm{~J}_{\mathrm{HH}} 0.9 \mathrm{~Hz}, 1 \mathrm{H}\right.$, $H$-C-P), $1.29\left(\mathrm{t},{ }^{3} \mathrm{~J}_{\mathrm{HH}} 7.0 \mathrm{~Hz}, 3 \mathrm{H}, \mathrm{CH}_{3^{-}}\right), 1.07\left(\mathrm{t},{ }^{3} \mathrm{~J}_{\mathrm{HH}} 7.0 \mathrm{~Hz}, 3 \mathrm{H}, \mathrm{CH}_{3^{-}}\right) ; \delta_{\mathrm{C}}\left(176 \mathrm{MHz}\right.$, acetone-d6) 163.4 (d, ${ }^{2} J_{\mathrm{PC}} 5.4$ $\mathrm{Hz}$, O-C(O)), $151.5\left(C_{A r}\right), 139.6\left(C_{A r}\right), 130.2\left(C_{A r}\right), 129.8\left(-\mathrm{C}-C_{A r} \mathrm{H}-\mathrm{CCl}\right), 129.6\left(\mathrm{CH}-C_{A r} \mathrm{H}-\mathrm{CCN}\right), 126.4\left(C_{A r}\right), 126.0$ $\left(C_{A r} \mathrm{H}\right), 125.8\left(-C_{A r} \mathrm{H}-\mathrm{NH}-\right), 124.7\left(\mathrm{C}-C_{A r} \mathrm{H}-\mathrm{CCN}\right), 120.9\left(C_{A r}\right), 119.3\left(-\mathrm{C}-C_{A r} \mathrm{H}-\mathrm{CH}-\mathrm{CCl}\right), 117.6\left(\mathrm{~d},{ }^{3} J_{\mathrm{PC}} 17.9 \mathrm{~Hz},-C_{A r}\right.$ $\mathrm{C}_{\mathrm{Ar}} \mathrm{H}-\mathrm{N}$ ), 114.1 (C- $\left.\mathrm{C}_{A r} \mathrm{H}-\mathrm{CCl}\right), 103.6\left(C_{A r}\right), 64.0$ (d, $\left.{ }^{2} J_{\mathrm{PC}} 6.4 \mathrm{~Hz}, \mathrm{CH}_{3}-\mathrm{CH}_{2}-\mathrm{OP}\right), 63.7$ (d, $\left.{ }^{2} J_{\mathrm{PC}} 6.6 \mathrm{~Hz}, \mathrm{CH}_{3}-\mathrm{CH}_{2}-\mathrm{OP}\right), 47.9$ (d, $\left.{ }^{1} J_{P C} 123.5 \mathrm{~Hz},-C H-P\right), 35.0\left(d,{ }^{3} J_{P C} 3.2 \mathrm{~Hz},-\mathrm{C}_{\mathrm{Ar}}-\mathrm{CH}-\mathrm{C}_{\mathrm{Ar}}\right.$ ), $16.5\left(\mathrm{~d},{ }^{3} \mathrm{~J}_{\mathrm{PC}} 6.1 \mathrm{~Hz}, \mathrm{CH}_{3}-\mathrm{CH}_{2}-\mathrm{OP}\right), 16.3\left(\mathrm{~d},{ }^{3} \mathrm{~J}_{\mathrm{PC}} 6.1 \mathrm{~Hz}\right.$, $\left.\mathrm{CH}_{3}-\mathrm{CH}_{2}-\mathrm{OP}\right) ; \delta_{\mathrm{P}}(283.3 \mathrm{MHz}$, acetone-d6) $17.6 \mathrm{ppm}$. 
General procedure for the synthesis 3-methylene-4-(indol-3-yl)-3,4-dihydrocoumarins (4a-f). A mixture of a 3-diethoxyphosphoryl-4-(1H-indol-3-yl)-3,4-dihydrocoumarin (3a-f) $(0.5 \mathrm{mmol})$ and $\mathrm{K}_{2} \mathrm{CO}_{3}(0.207 \mathrm{~g}, 1.5 \mathrm{mmol})$ in THF $(5 \mathrm{~mL})$ was stirred at $0{ }^{\circ} \mathrm{C}$ for $15 \mathrm{~min}$. Then, aq formaldehyde $(40 \%, 0.20 \mathrm{~mL})$ was added and resulting suspension was stirred at $20^{\circ} \mathrm{C}$ for an additional $3 \mathrm{~h}$. The mixture was then concentrated in vacuo and the solid residue was extracted with $\mathrm{CH}_{2} \mathrm{Cl}_{2}(3 \times 20 \mathrm{~mL})$. The combined organic layers were dried over $\mathrm{MgSO}_{4}$ and evaporated. The oily residue was subjected for column chromatography on silica gel using $\mathrm{CH}_{2} \mathrm{Cl}_{2} / \mathrm{Me}_{2} \mathrm{CO}$ (10:1) as eluent $\left(R_{F} \sim 0.8\right)$ to afford desired products (4a-f).

4-(1H-Indol-3-yl)-3-methylenechroman-2-one (4a). Colorless foam; Anal. calcd for $\mathrm{C}_{18} \mathrm{H}_{13} \mathrm{NO}_{2}: \mathrm{C}, 78.53 ; \mathrm{H}$, 4.76; N, 5.09; Found C, 78.6; H, 4.7; N, 5.1; IR(ATR): 3333, 1724, 1454, 1278, 1235, 1219, 1153, 1141, 1105, $1094,972,756,740,668,639,597,552,430 \mathrm{~cm}^{-1} ; \delta_{\mathrm{H}}\left(700 \mathrm{MHz}, \mathrm{CDCl}_{3}\right) 8.13$ (bs, $\left.1 \mathrm{H}, \mathrm{NH}\right), 7.05-7.42(\mathrm{~m}, 8 \mathrm{H}, \mathrm{H}-$ $\left.\mathrm{C}_{\mathrm{Ar}}\right), 6.94\left(\mathrm{bd},{ }^{3} J_{\mathrm{HH}} 2.5 \mathrm{~Hz}, 1 \mathrm{H},-\mathrm{CH}-\mathrm{NH}-\right), 6.41\left(\mathrm{dd},{ }^{2} J_{\mathrm{HH}} 1.7 \mathrm{~Hz},{ }^{4} J_{\mathrm{HH}} 0.9 \mathrm{~Hz}, 1 \mathrm{H},=\mathrm{CHH}\right), 5.72\left(\mathrm{dd},{ }^{2} J_{\mathrm{HH}} 1.7 \mathrm{~Hz},{ }^{4} J_{\mathrm{HH}}\right.$ $0.9 \mathrm{~Hz}, 1 \mathrm{H},=\mathrm{CHH}), 5.25$ (bs, $\left.1 \mathrm{H},-\mathrm{CH}-\mathrm{C}=\mathrm{CH}_{2}\right) ; \delta_{\mathrm{C}}\left(176 \mathrm{MHz}, \mathrm{CDCl}_{3}\right) 163.9(\mathrm{O}-\mathrm{C}(\mathrm{O})), 151.8\left(C_{A r}\right), 137.1\left(C_{A r}\right), 136.1$ $\left(=C_{A r}<\right), 128.7\left(C_{A r} H\right), 128.6\left(=C H_{2}\right), 128.4\left(C_{A r} H\right), 125.5\left(C_{A r}\right), 125.2\left(C_{A r}\right), 124.9\left(C_{A r} \mathrm{H}\right), 123.7\left(C_{A r} H\right), 122.7\left(C_{A r} \mathrm{H}\right)$, $119.8\left(C_{A r} \mathrm{H}\right), 117.2\left(C_{A r} \mathrm{H}\right), 114.1\left(-C_{A r}-C_{A r} \mathrm{H}-\mathrm{N}\right), 111.7\left(C_{A r} \mathrm{H}\right), 40.3\left(-C_{A r}-C H-C_{A r}\right)$.

4-(1H-Indol-3-yl)-8-methoxy-3-methylenechroman-2-one (4b). Colorless foam; Anal. calcd for $\mathrm{C}_{19} \mathrm{H}_{15} \mathrm{NO}_{3}$ : $\mathrm{C}$, 74.74; H, 4.95; N, 4.59; Found C, 74.9; H, 5.0; N, 4.6; IR(ATR): 3403, 1738, 1481, 1456, 1285, 1271, 1186, 1123, $1089,1062,955,769,740 \mathrm{~cm}^{-1} ; \delta_{\mathrm{H}}\left(700 \mathrm{MHz} \mathrm{CDCl}_{3}\right) 8.19(\mathrm{bs}, 1 \mathrm{H}, \mathrm{NH}), 7.04-7.40\left(\mathrm{~m}, 4 \mathrm{H}, \mathrm{H}_{\mathrm{ind}}\right), 6.99\left(\mathrm{t}^{3} \mathrm{~J}_{\mathrm{HH}} 8.0\right.$ $\mathrm{Hz}, 1 \mathrm{H},-\mathrm{CH}-\mathrm{CH}-\mathrm{C}-\mathrm{OMe}), 6.93\left(\mathrm{bd},{ }^{3} \mathrm{~J}_{\mathrm{HH}} 2.4 \mathrm{~Hz}, 1 \mathrm{H},-\mathrm{CH}-\mathrm{NH}-\right), 6.88\left(\mathrm{dd},{ }^{3} \mathrm{~J}_{\mathrm{HH}} 8.0 \mathrm{~Hz},{ }^{4} \mathrm{~J}_{\mathrm{HH}} 1.3 \mathrm{~Hz}, 1 \mathrm{H},-\mathrm{CH}-\mathrm{C}-\mathrm{C}-\mathrm{O}\right)$, $6.68\left(\mathrm{dd},{ }^{3} J_{\mathrm{HH}} 8.0 \mathrm{~Hz},{ }^{4} J_{\mathrm{HH}}=1.3 \mathrm{~Hz}, 1 \mathrm{H},-\mathrm{CH}-\mathrm{C}-\mathrm{OMe}\right), 6.39$ (dd, $\left.{ }^{2} J_{\mathrm{HH}} 1.7 \mathrm{~Hz},{ }^{4} J_{\mathrm{HH}} 0.9 \mathrm{~Hz}, 1 \mathrm{H},=\mathrm{CHH}\right), 5.72\left(\mathrm{dd},{ }^{2} J_{\mathrm{HH}}\right.$ $\left.1.7 \mathrm{~Hz},{ }^{4} J_{\mathrm{HH}} 0.9 \mathrm{~Hz}, 1 \mathrm{H},=\mathrm{CHH}\right), 5.23$ (bs, $\left.1 \mathrm{H},-\mathrm{CH}-\mathrm{C}=\mathrm{CH}_{2}\right), 3.92\left(\mathrm{~s}, 3 \mathrm{H},-\mathrm{OCH}_{3}\right) ; \delta_{\mathrm{C}}\left(176 \mathrm{MHz}, \mathrm{CDCl}_{3}\right) 163.3(\mathrm{O}-\mathrm{C}(\mathrm{O}))$, $147.9\left(C_{A r}\right), 140.2\left(C_{A r}\right), 137.1\left(C_{A r}\right), 136.0(=C<), 128.4\left(=C H_{2}\right), 126.4\left(C_{A r}\right), 125.5\left(C_{A r}\right), 124.7\left(C_{A r} H\right), 123.6\left(C_{A r} H\right)$, $122.6\left(C_{A r} \mathrm{H}\right), 119.9\left(C_{A r} \mathrm{H}\right), 119.8\left(2 \times C_{A r} \mathrm{H}\right), 114.1\left(C_{A r}\right), 111.7\left(C_{A r} \mathrm{H}\right), 111.4\left(C_{A r} \mathrm{H}\right), 56.3\left(-0 \mathrm{CH}_{3}\right), 40.5\left(-\mathrm{C}_{A r}-\mathrm{CH}-\right.$ $\mathrm{C}_{\mathrm{Ar}}$ ).

6-Chloro-4-(1H-indol-3-yl)-3-methylenechroman-2-one (4c). Colorless foam; Anal. calcd for $\mathrm{C}_{18} \mathrm{H}_{12} \mathrm{ClNO}_{2}: \mathrm{C}_{\text {, }}$ 69.80; H, 3.90; N, 4.52; Found C, 70.0.; H, 3.9; N, 4.5; IR(ATR): 3402, 1736, 1477, 1457, 1409, 1296, 1232, 1178, $1133,1105,1086,817,740,526,425 \mathrm{~cm}^{-1} ; \delta_{\mathrm{H}}\left(700 \mathrm{MHz}_{\mathrm{C}} \mathrm{CDCl}_{3}\right) 8.23$ (bs, $\left.1 \mathrm{H}, \mathrm{NH}\right), 7.04-7.43$ (m, 7H, H aromat.), $6.97\left(\mathrm{bd},{ }^{3} J_{\mathrm{HH}} 2.5 \mathrm{~Hz}, 1 \mathrm{H},-\mathrm{CH}-\mathrm{NH}-\right), 6.44\left(\mathrm{dd},{ }^{2} J_{\mathrm{HH}} 1.8 \mathrm{~Hz},{ }^{4} J_{\mathrm{HH}} 0.7 \mathrm{~Hz}, 1 \mathrm{H},=\mathrm{CHH}\right), 5.72\left(\mathrm{dd},{ }^{2} J_{\mathrm{HH}} 1.8 \mathrm{~Hz},{ }^{4} J_{\mathrm{HH}} 0.7 \mathrm{~Hz}\right.$, $1 \mathrm{H},=\mathrm{CHH}), 5.21$ (bs, $\left.1 \mathrm{H},-\mathrm{CH}-\mathrm{C}=\mathrm{CH}_{2}\right) ; \delta_{\mathrm{C}}\left(176 \mathrm{MHz}, \mathrm{CDCl}_{3}\right) 163.2(\mathrm{O}-\mathrm{C}(\mathrm{O})), 149.4\left(C_{A r}\right), 137.1\left(C_{A r}\right), 135.2(=C<)$, $130.0\left(C_{A r}\right), 129.6\left(C_{A r} \mathrm{H}\right), 128.8\left(=C_{2}\right), 128.2\left(C_{A r} \mathrm{H}\right), 127.0\left(C_{A r}\right), 125.2\left(C_{A r}\right), 123.7\left(C_{A r} \mathrm{H}\right), 122.9\left(C_{A r} \mathrm{H}\right), 120.2$ $\left(C_{A r} \mathrm{H}\right), 119.7\left(C_{A r} \mathrm{H}\right), 118.6\left(C_{A r} \mathrm{H}\right), 113.4\left(C_{A r}\right), 111.8\left(C_{A r} \mathrm{H}\right), 40.2\left(-C_{A r}-C H-C_{A r}\right)$.

4-(5-Methoxy-1H-indol-3-yl)-3-methylenechroman-2-one (4d). Colorless foam; Anal. calcd for $\mathrm{C}_{19} \mathrm{H}_{15} \mathrm{NO}_{3}: \mathrm{C}_{\text {, }}$ 74.74; H, 4.95; N, 4.59; Found C, 74.6; H, 5.0; N, 4.6; IR(ATR): 3304, 1731, 1483, 1452, 1251, 1230, 1169, 1141, $1099,1061,948,801,753,657,634,615,562 \mathrm{~cm}^{-1} ; \delta_{\mathrm{H}}\left(700 \mathrm{MHz}, \mathrm{CDCl}_{3}\right) 8.03$ (bs, $\left.1 \mathrm{H}, \mathrm{NH}\right), 7.27-7.30(\mathrm{~m}, 2 \mathrm{H}, \mathrm{H}-$ $\left.\mathrm{C}_{\mathrm{Ar}}\right), 7.15\left(\mathrm{dd},{ }^{3} \mathrm{~J}_{\mathrm{HH}} 8.1 \mathrm{~Hz},{ }^{5} \mathrm{~J}_{\mathrm{HH}} 1.3 \mathrm{~Hz}, 1 \mathrm{H},-\mathrm{CH}-\mathrm{CH}-\mathrm{C}-\mathrm{OMe}\right), 7.04-7.11\left(\mathrm{~m}, 2 \mathrm{H}, \mathrm{H}-\mathrm{C}_{\mathrm{Ar}}\right), 6.93\left(\mathrm{bd},{ }^{3} \mathrm{~J}_{\mathrm{HH}} 2.4 \mathrm{~Hz}, 1 \mathrm{H},-\right.$ $\mathrm{CH}-\mathrm{NH}-$ ) $, 6.87\left(\mathrm{dd},{ }^{3} J_{\mathrm{HH}} 8.8 \mathrm{~Hz},{ }^{4} J_{\mathrm{HH}}=2.5 \mathrm{~Hz}, 1 \mathrm{H},-\mathrm{CH}-\mathrm{CH}-\mathrm{C}-\mathrm{OMe}\right), 6.73\left(\mathrm{~d},{ }^{4} J_{\mathrm{HH}} 2.5 \mathrm{~Hz}, 1 \mathrm{H},-\mathrm{C}-\mathrm{CH}-\mathrm{C}-\mathrm{OMe}\right), 6.41$ (dd, $\left.{ }^{2} J_{\mathrm{HH}} 1.7 \mathrm{~Hz},{ }^{4} J_{\mathrm{HH}} 0.9 \mathrm{~Hz}, 1 \mathrm{H},=\mathrm{CHH}\right), 5.71\left(\mathrm{dd},{ }^{2} J_{\mathrm{HH}} 1.7 \mathrm{~Hz},{ }^{4} J_{\mathrm{HH}} 0.9 \mathrm{~Hz}, 1 \mathrm{H},=\mathrm{CHH}\right), 5.21\left(\mathrm{bs}, 1 \mathrm{H},-\mathrm{CH}-\mathrm{C}=\mathrm{CH}_{2}\right)$, $3.74\left(\mathrm{~s}, 3 \mathrm{H},-\mathrm{CH}_{3}\right) ; \delta_{\mathrm{C}}\left(176 \mathrm{MHz}, \mathrm{CDCl}_{3}\right) 164.0(\mathrm{O}-\mathrm{C}(\mathrm{O})), 154.2\left(C_{A r}\right), 150.9\left(C_{A r}\right), 136.0(=C<), 132.2\left(C_{A r}\right), 128.7$ $\left(C_{A r} \mathrm{H}\right), 128.5\left(=\mathrm{CH}_{2}\right), 128.4\left(C_{A r} \mathrm{H}\right), 126.0\left(C_{A r}\right), 125.1\left(C_{A r}\right), 124.9\left(C_{A r} \mathrm{H}\right), 124.5\left(C_{A r} \mathrm{H}\right), 117.2\left(C_{A r} \mathrm{H}\right), 113.6\left(C_{A r}\right)$, $112.6\left(C_{A r} H\right), 112.4\left(C_{A r} H\right), 102.0\left(C_{A r} H\right), 56.0\left(-\mathrm{OCH}_{3}\right), 40.3\left(-\mathrm{C}_{\mathrm{Ar}}-\mathrm{CH}-\mathrm{C}_{\mathrm{Ar}}\right)$.

8-Methoxy-4-(5-methoxy-1H-indol-3-yl)-3-methylenechroman-2-one (4e). Colorless foam; Anal. calcd for $\mathrm{C}_{20} \mathrm{H}_{17} \mathrm{NO}_{4}$ : C, 71.63; H, 5.11; N, 4.18; Found C, 71.8; H, 5.1; N, 4.1; IR(ATR): 3442, 1738, 1484, 1444, 1285, $1189,1132,1093,801,795,767,604,499 \mathrm{~cm}^{-1} ; \delta_{\mathrm{H}}\left(700 \mathrm{MHz}, \mathrm{CDCl}_{3}\right) 8.03(\mathrm{bs}, 1 \mathrm{H}, \mathrm{NH}), 7.28\left(\mathrm{dd},{ }^{3} \mathrm{JHH}_{\mathrm{HH}} 8.8 \mathrm{~Hz}\right.$, $\left.{ }^{5} J_{\mathrm{HH}} 0.5 \mathrm{~Hz}, 1 \mathrm{H},-\mathrm{N}-\mathrm{C}-\mathrm{CH}-\mathrm{CH}-\mathrm{C}-\mathrm{OMe}\right), 7.00\left(\mathrm{t},{ }^{3} \mathrm{~J}_{\mathrm{HH}} 8.0 \mathrm{~Hz}, 1 \mathrm{H},-\mathrm{CH}-\mathrm{CH}-\mathrm{CH}-\right), 6.93$ (bd, ${ }^{3} \mathrm{~J}_{\mathrm{HH}} 2.4 \mathrm{~Hz}, 1 \mathrm{H},-\mathrm{CH}-\mathrm{NH}-$ ), $6.88\left(\mathrm{dd},{ }^{3} J_{\mathrm{HH}} 8.0 \mathrm{~Hz},{ }^{4} J_{\mathrm{HH}} 1.3 \mathrm{~Hz}, 1 \mathrm{H},-\mathrm{CH}-\mathrm{CH}-\mathrm{CH}-\mathrm{C}-\mathrm{OMe}\right), 6.86$ (dd, ${ }^{3} J_{\mathrm{HH}} 8.8 \mathrm{~Hz},{ }^{4} J_{\mathrm{HH}} 2.5 \mathrm{~Hz}, 1 \mathrm{H},-\mathrm{C}-\mathrm{CH}-\mathrm{CH}-\mathrm{C}-$ 
OMe), 6.76 (d, ${ }^{4} J_{\mathrm{HH}} 2.5 \mathrm{~Hz}, 1 \mathrm{H},-\mathrm{C}-\mathrm{CH}-\mathrm{C}-\mathrm{OMe}$ ), 6.69 (ddd, ${ }^{3} \mathrm{~J}_{\mathrm{HH}} 8.0 \mathrm{~Hz},{ }^{4} J_{\mathrm{HH}} 1.3 \mathrm{~Hz},{ }^{5} J_{\mathrm{HH}} 0.8 \mathrm{~Hz}, 1 \mathrm{H},-\mathrm{CH}-\mathrm{CH}-\mathrm{CH}-\mathrm{C}-$ OMe), $6.40\left(\mathrm{dd},{ }^{2} J_{\mathrm{HH}} 1.7 \mathrm{~Hz},{ }^{4} J_{\mathrm{HH}}=0.9 \mathrm{~Hz}, 1 \mathrm{H},=\mathrm{CHH}\right), 5.70\left(\mathrm{dd},{ }^{2} J_{\mathrm{HH}} 1.7 \mathrm{~Hz},{ }^{4} J_{\mathrm{HH}} 0.9 \mathrm{~Hz}, 1 \mathrm{H},=\mathrm{CHH}\right), 5.19$ (bs, $1 \mathrm{H},-$ $\left.\mathrm{CH}-\mathrm{C}=\mathrm{CH}_{2}\right), 3.93\left(\mathrm{~s}, 3 \mathrm{H},-\mathrm{CH}_{3}\right), 3.75\left(\mathrm{~s}, 3 \mathrm{H},-\mathrm{CH}_{3}\right) ; \delta_{\mathrm{C}}\left(176 \mathrm{MHz}, \mathrm{CDCl}_{3}\right) 163.4(\mathrm{O}-\mathrm{C}(\mathrm{O})), 154.1\left(C_{A r}\right), 147.9\left(C_{A r}\right)$, $140.2\left(C_{A r}\right), 135.9(=C<), 132.2\left(C_{A r}\right), 128.7\left(C_{A r} \mathrm{H}\right), 128.3\left(=C_{2}\right), 126.3\left(C_{A r}\right), 126.0\left(C_{A r}\right), 124.7\left(C_{A r} H\right), 124.4$ $\left(C_{A r} H\right), 119.7\left(C_{A r} H\right), 113.5\left(C_{A r}\right), 112.5\left(C_{A r} H\right), 112.4\left(C_{A r} H\right), 111.4\left(C_{A r} H\right), 101.9\left(C_{A r} H\right), 56.3\left(-O C H_{3}\right), 56.0(-$ $\left.\mathrm{OCH}_{3}\right), 40.5\left(-\mathrm{C}_{\mathrm{Ar}}-\mathrm{CH}-\mathrm{C}_{\mathrm{Ar}}\right)$.

6-Chloro-4-(5-methoxy-1H-indol-3-yl)-3-methylenechroman-2-one (4f). Colorless foam; Anal. calcd for $\mathrm{C}_{19} \mathrm{H}_{14} \mathrm{CINO}_{3}$ : C, 67.16; H, 4.15; N, 4.12; Found C, 67.4; H, 4.1; N, 4.1; IR(ATR): 3404, 2996, 2939, 2899, 2830, $1738,1478,1217,1172,1131,1106,1083,1049,1024,800,530 \mathrm{~cm}^{-1} ; \delta_{H}\left(700 \mathrm{MHz} \mathrm{CDCl}_{3}\right) 8.06$ (bs, $\left.1 \mathrm{H}, \mathrm{NH}\right)$, 7.31 (dd, ${ }^{3} J_{\mathrm{HH}} 8.8 \mathrm{~Hz},{ }^{5} J_{\mathrm{HH}} 0.5 \mathrm{~Hz}, 1 \mathrm{H},-\mathrm{N}-\mathrm{C}-\mathrm{CH}-\mathrm{CH}-\mathrm{C}-\mathrm{OMe}$ ), 7.24 (ddd, ${ }^{3} J_{\mathrm{HH}} 8.7 \mathrm{~Hz},{ }^{4} J_{\mathrm{HH}} 2.5 \mathrm{~Hz},{ }^{5} J_{\mathrm{HH}} 0.6 \mathrm{~Hz}, 1 \mathrm{H},-$ $\mathrm{CCl}-\mathrm{CH}-\mathrm{CH}-$ ), 7.09 (d, ${ }^{3} \mathrm{~J}_{\mathrm{HH}} 8.7 \mathrm{~Hz}, 1 \mathrm{H},-\mathrm{CCl}-\mathrm{CH}-\mathrm{CH}-$ ), 7.06 (dd, ${ }^{4} J_{\mathrm{HH}} 2.5 \mathrm{~Hz},{ }^{5} J_{\mathrm{HH}} 0.9 \mathrm{~Hz}, 1 \mathrm{H},-\mathrm{C}-\mathrm{CCl}-\mathrm{CH}-$ ), 6.97 (bd, $\left.{ }^{3} J_{\mathrm{HH}} 2.4 \mathrm{~Hz}, 1 \mathrm{H},-\mathrm{CH}-\mathrm{NH}-\right), 6.89$ (dd, $\left.{ }^{3} \mathrm{~J}_{\mathrm{HH}} 8.8 \mathrm{~Hz},{ }^{4} \mathrm{~J}_{\mathrm{HH}} 2.5 \mathrm{~Hz}, 1 \mathrm{H},-\mathrm{C}-\mathrm{CH}-\mathrm{CH}-\mathrm{C}-\mathrm{OMe}\right), 6.71\left(\mathrm{~d},{ }^{4} \mathrm{~J}_{\mathrm{HH}} 2.5 \mathrm{~Hz}, 1 \mathrm{H},-\mathrm{C}-\mathrm{CH}-\right.$ C-OMe), $6.45\left(\mathrm{dd},{ }^{2} J_{\mathrm{HH}} 1.9 \mathrm{~Hz},{ }^{4} J_{\mathrm{HH}} 0.8 \mathrm{~Hz}, 1 \mathrm{H},=\mathrm{CHH}\right), 5.71\left(\mathrm{dd},{ }^{2} J_{\mathrm{HH}} 1.9 \mathrm{~Hz},{ }^{4} J_{\mathrm{HH}} 0.8 \mathrm{~Hz}, 1 \mathrm{H},=\mathrm{CHH}\right), 5.18$ (bs, $1 \mathrm{H},-$ $\left.\mathrm{CH}-\mathrm{C}=\mathrm{CH}_{2}\right), 3.76\left(\mathrm{~s}, 3 \mathrm{H},-\mathrm{CH}_{3}\right) ; \delta_{\mathrm{C}}\left(176 \mathrm{MHz}, \mathrm{CDCl}_{3}\right) 163.3(\mathrm{O}-\mathrm{C}(\mathrm{O})), 154.3\left(C_{A r}\right), 149.4\left(C_{A r}\right), 140.2\left(C_{A r}\right), 135.9$ $132.2\left(C_{A r}\right), 128.7\left(C_{A r} \mathrm{H}\right), 135.0(=C<), 132.2\left(C_{A r}\right), 130.0\left(C_{A r}\right), 129.4\left(=C_{2}\right), 128.8\left(C_{A r} \mathrm{H}\right), 128.2\left(C_{A r} \mathrm{H}\right), 126.9$ $\left(C_{A r}\right), 125.7\left(C_{A r}\right), 124.5\left(C_{A r} \mathrm{H}\right), 118.6\left(C_{A r} \mathrm{H}\right), 112.7\left(C_{A r} \mathrm{H}\right), 112.5\left(C_{A r} \mathrm{H}\right), 101.8\left(C_{A r} \mathrm{H}\right), 56.0\left(-\mathrm{OCH}_{3}\right), 40.25\left(-C_{A r}\right.$ $\left.\mathrm{CH}-\mathrm{C}_{\mathrm{Ar}}\right)$.

Diethyl (8-hydroxy-2-oxo-2H-chromen-3-yl)phosphonate (7). To a solution of 2,3-dihydroxybenzaldehyde (6.9 $\mathrm{g}, 50 \mathrm{mmol}) 5$ and triethyl phosphonoacetate $6(11.2 \mathrm{~g}, 50 \mathrm{mmol})$ in toluene $(100 \mathrm{~mL})$ piperidine $(0.5 \mathrm{~mL})$ and acetic acid $(1.0 \mathrm{~mL})$ were added. The solution was then heated at reflux under a Dean-Stark trap until the starting materials were consumed (ca. $15 \mathrm{~h}, \mathrm{TLC}$ and ${ }^{31} \mathrm{P}$ NMR monitoring). After evaporation of the solvent the residue was purified by chromatography using $\mathrm{CH}_{2} \mathrm{Cl}_{2} / \mathrm{MeOH}, 15: 1$, as eluent $\left(R_{F} \sim 0.6\right)$ to yield desired phosphonate 7 (8.05 g, 54\%). Colorless crystals, mp $126-128{ }^{\circ} \mathrm{C}$; Anal. calcd for $\mathrm{C}_{13} \mathrm{H}_{15} \mathrm{O}_{6} \mathrm{P}: \mathrm{C}, 52.36 ; \mathrm{H}, 5.07$; Found C, 52.3; H, 5.1; IR(ATR): 3147, 2986, 2908, 1717, 1577, 1465, 1220, 1166, 1047, 1013, 994, 974, 957, $764,632,499 \mathrm{~cm}^{-1} ; \delta_{\mathrm{H}}(700 \mathrm{MHz}$, acetone-d6) 9.22 (bs, $1 \mathrm{H},-\mathrm{OH}), 8.54\left(\mathrm{~d},{ }^{2} J_{\mathrm{PH}} 17.3 \mathrm{~Hz}, 1 \mathrm{H}, \mathrm{H}-\mathrm{CP}\right), 7.32$ (dd, ${ }^{3} J_{\mathrm{HH}}$ $\left.7.3 \mathrm{~Hz},{ }^{4} J_{\mathrm{HH}} 1.4 \mathrm{~Hz}, 1 \mathrm{H}, \mathrm{C}-\mathrm{CH}-\mathrm{CH}-\right), 7.28\left(\mathrm{dd},{ }^{3} J_{\mathrm{HH}} 7.3 \mathrm{~Hz},{ }^{4} J_{\mathrm{HH}} 1.4 \mathrm{~Hz}, 1 \mathrm{H}, \mathrm{CH}-\mathrm{C}(\mathrm{OH})-\right), 7.24\left(\mathrm{t},{ }^{3} J_{\mathrm{HH}} 7.3 \mathrm{~Hz}, 1 \mathrm{H},-\mathrm{CH}-\right.$ $\mathrm{CH}-\mathrm{CH}-)$, 4.17-4.30 (m, 4H, $\left.-\mathrm{CH}_{2}-\right), 1.32\left(\mathrm{t},{ }^{3} J_{\mathrm{HH}} 7.1,6 \mathrm{H},-\mathrm{CH}_{3}\right) ; \delta_{\mathrm{C}}\left(700 \mathrm{MHz}\right.$, acetone-d6) $158.2\left(\mathrm{~d},{ }^{2} J_{\mathrm{PC}} 15.1 \mathrm{~Hz}, 0-\right.$ $C(\mathrm{O})), 154.4\left(\mathrm{~d},{ }^{2} J_{\mathrm{PC}} 6.1 \mathrm{~Hz}, \mathrm{HC} C_{A r}-C_{A r} \mathrm{P}\right), 145.4\left(C_{A r}\right), 144.6\left(C_{A r}\right), 125.4\left(C_{A r} \mathrm{H}\right), 121.4\left(C_{A r} \mathrm{H}\right), 121.2\left(C_{A r} \mathrm{H}\right), 119.7(\mathrm{~d}$, $\left.{ }^{3} J_{P C} 14.0 \mathrm{~Hz},-C_{A r}\right), 118.9\left(d,{ }^{1} J_{P C} 195.0 \mathrm{~Hz},-C_{A r}\right), 63.4\left(d,{ }^{2} J_{P C} 5.6 \mathrm{~Hz}, \mathrm{CH}_{3}-C_{2}-\mathrm{OP}\right), 16.7\left(\mathrm{~d},{ }^{3} \mathrm{JPC}_{\mathrm{PC}} 6.2 \mathrm{~Hz}, \mathrm{CH}_{3}-\mathrm{CH}_{2^{-}}\right.$ $\mathrm{OP}) ; \delta_{\mathrm{P}}\left(283.3 \mathrm{MHz}, \mathrm{CDCl}_{3}\right) 12.5 \mathrm{ppm}$.

Diethyl (8-((tert-butyldimethylsilyl)oxy)-2-oxo-2H-chromen-3-yl)phosphonate (8). To a stirred solution of diethyl (8-hydroxy-2-oxo-2H-chromen-3-yl)phosphonate 7 (0.895 g, $3.0 \mathrm{mmol})$ and imidazole $(0.408 \mathrm{~g}, 6.0$ $\mathrm{mmol}$ ) in $\mathrm{CH}_{2} \mathrm{Cl}_{2}(15 \mathrm{~mL})$, tert-butyldimethylsilyl chloride (TBDMS-Cl) $(0.497 \mathrm{~g}, 3.3 \mathrm{mmol})$ was added in one portion and the resulting mixture was stirred at $\mathrm{rt}$ for $24 \mathrm{~h}$. Then solution was transferred into a separatory funnel and successively washed with $1 \mathrm{M}$ solution of citric acid $(15 \mathrm{~mL})$ and $1 \mathrm{M}$ solution of $\mathrm{NaHCO}_{3}(15 \mathrm{~mL})$. The organic layer was dried over $\mathrm{MgSO}_{4}$ and evaporated. The crude product was purified by chromatography on silica gel using $\mathrm{CH}_{2} \mathrm{Cl}_{2} / \mathrm{Me}_{2} \mathrm{CO}(10: 1)$ as eluent $\left(R_{F} \sim 0.7\right)$ to afford the desired silyl-protected coumarin 8 (1.126 g, 91\%). Colorless crystals, mp 81-83 ${ }^{\circ} \mathrm{C}$; Anal. calcd for $\mathrm{C}_{19} \mathrm{H}_{29} \mathrm{O}_{6} \mathrm{PSi}$ : $\mathrm{C}, 55.32 ; \mathrm{H}, 7.09$; Found $\mathrm{C}, 55.2 ; \mathrm{H}$, 7.1; IR(ATR): 3363, 3186, 1767, 1666, 1569, 1384, 1367, 1309, 1139, 1124, 1034, 749, 734, 539, $505 \mathrm{~cm}^{-1} ; \delta_{H}$ $\left(700 \mathrm{MHz}, \mathrm{CDCl}_{3}\right) 8.46\left(\mathrm{~d},{ }^{3} \mathrm{~J}_{\mathrm{PH}} 17.2 \mathrm{~Hz}, 1 \mathrm{H}, \mathrm{HC}_{\mathrm{Ar}}-\mathrm{C}_{\mathrm{Ar}} \mathrm{P}\right), 7.12-7.19\left(\mathrm{~m}, 3 \mathrm{H}, \mathrm{C}_{\mathrm{Ar}} \mathrm{H}-\mathrm{C}_{\mathrm{Ar}}\right), 4.20-4.33\left(\mathrm{~m}, 4 \mathrm{H},-\mathrm{CH}_{2^{-}}\right), 1.37$ $\left(\mathrm{t},{ }^{3} J_{\mathrm{HH}} 7.1,{ }^{4} J_{\mathrm{PH}} 0.5 \mathrm{~Hz}, 6 \mathrm{H},-\mathrm{CH}_{2}-\mathrm{CH}_{3}\right), 1.03\left(\mathrm{~s}, 9 \mathrm{H},{ }^{\mathrm{t}} \mathrm{Bu}\right), 0.25\left(\mathrm{~s}, 6 \mathrm{H}, \mathrm{Si}\left(\mathrm{CH}_{3}\right)_{2}\right) ; \delta_{\mathrm{C}}\left(176 \mathrm{MHz}, \mathrm{CDCl}_{3}\right) 157.9\left(\mathrm{~d},{ }^{2} J_{\mathrm{PC}}\right.$ $14.5 \mathrm{~Hz}, \mathrm{O}-\mathrm{C}(\mathrm{O})), 153.7\left(\mathrm{~d},{ }^{2} J_{\mathrm{PC}} 6.5 \mathrm{~Hz}, \mathrm{HC} C_{A r}-\mathrm{C}_{\mathrm{Ar}} \mathrm{P}\right), 146.9\left(C_{A r}\right), 143.4\left(C_{A r}\right), 125.4\left(C_{A r} \mathrm{H}\right), 124.9\left(C_{A r} \mathrm{H}\right), 121.8$ $\left(C_{A r} H\right), 119.2\left(d,{ }^{3} J_{P C} 14.1 \mathrm{~Hz},-C_{A r}\right), 118.0\left(\mathrm{~d},{ }^{1} J_{\mathrm{PC}} 196.4 \mathrm{~Hz},-C_{A r}\right), 63.6\left(\mathrm{~d},{ }^{2} J_{\mathrm{PC}} 6.1 \mathrm{~Hz}, \mathrm{CH}_{3}-C_{2}-\mathrm{OP}\right), 25.7(-\mathrm{C}-$ $\left.\left(\mathrm{CH}_{3}\right)_{3}\right), 18.5\left(-\mathrm{C}-\left(\mathrm{CH}_{3}\right)_{3}\right), 16.5\left(\mathrm{~d},{ }^{3}{ }_{\mathrm{PC}} 6.3 \mathrm{~Hz}, \mathrm{CH}_{3}-\mathrm{CH}_{2}-\mathrm{OP}\right),-4.3\left(-\mathrm{Si}\left(\mathrm{CH}_{3}\right)_{2}\right) ; \delta_{\mathrm{P}}\left(283.3 \mathrm{MHz}, \mathrm{CDCl}_{3}\right) 12.5 \mathrm{ppm}$. 
Diethyl ((3R* $\left.4 S^{*}\right)-8-(($ tert-butyldimethylsilyl)oxy)-4-(1H-indol-3-yl)-2-oxochroman-3-yl)phosphonate (9). To a stirred solution of the silyl-protected coumarin $8(0.412 \mathrm{~g}, 1.0 \mathrm{mmol})$ and indole $2 \mathrm{a}(0.176 \mathrm{~g}, 1.5 \mathrm{mmol})$ in $\mathrm{CH}_{2} \mathrm{Cl}_{2}(10 \mathrm{~mL}), 1,5,7$-triazabicyclo[4.4. 0]dec-5-ene $(0.278 \mathrm{~g}, 2.0 \mathrm{mmol})$ was added in one portion. Stirring was continued at $\mathrm{rt}$ for $24 \mathrm{~h}$. The resulting mixture was acidified with hydrochloric acid $(5 \%, 10 \mathrm{~mL})$ and separated. The aqueous layer was extracted with $\mathrm{CH}_{2} \mathrm{Cl}_{2}(3 \times 20 \mathrm{~mL})$. The combined organic layers were washed with brine, dried over $\mathrm{MgSO}_{4}$ and evaporated. The oily residue was subjected for column chromatography on silica gel using $\mathrm{CH}_{2} \mathrm{Cl}_{2} / \mathrm{MeOH}(20: 1)$ as eluent $\left(R_{F} \sim 0.60\right)$ to give pure phosphonate adduct 9 (0.281 g, 53\%). Colorless crystals, mp 172-174 ${ }^{\circ} \mathrm{C}$; Anal. calcd for $\mathrm{C}_{27} \mathrm{H}_{36} \mathrm{NO}_{6} \mathrm{PSi}$ : C, 61.23; $\mathrm{H}, 6.85 ; \mathrm{N}, 2.64$; Found $\mathrm{C}, 61.3 ; \mathrm{H}, 6.7 ; \mathrm{N}, 2.7$; IR(ATR): 3326, 2957, 2930, 2859, 1764, 1481, 1251, 1145, 1081, 1042, 1019, 867, 839, 825, 782, $738 \mathrm{~cm}^{-1} ; \delta_{\mathrm{H}}$ $\left(700 \mathrm{MHz} \mathrm{CDCl}_{3}\right) 8.09$ (bs, $\left.1 \mathrm{H}, \mathrm{NH}\right), 7.70\left(\mathrm{bd},{ }^{3} J_{\mathrm{HH}} 7.8 \mathrm{~Hz}, 1 \mathrm{H}, \mathrm{H}-\mathrm{C}_{\mathrm{Ar}}\right), 6.86-7.36\left(\mathrm{~m}, 6 \mathrm{H}, \mathrm{H}-\mathrm{C}_{\mathrm{Ar}}\right), 6.56\left(\mathrm{dd},{ }^{3} J_{\mathrm{HH}} 2.5\right.$ $\left.\mathrm{Hz},{ }^{4} J_{\mathrm{HH}} 0.9 \mathrm{~Hz}, 1 \mathrm{H},-\mathrm{CH}-\mathrm{NH}-\right), 5.08\left(\mathrm{bd},{ }^{3} \mathrm{~J}_{\mathrm{PH}} 12.8 \mathrm{~Hz}, 1 \mathrm{H},-\mathrm{CH}-\mathrm{C}_{\mathrm{Ar}}\right), 4.12-4.21\left(\mathrm{~m}, 2 \mathrm{H},-\mathrm{CH}_{2}-\right), 3.90-3.97$ and 3.65$3.72\left(\mathrm{~m}, 2 \mathrm{H},-\mathrm{CH}_{2^{-}}\right), 3.83\left(\mathrm{dd},{ }^{2} \mathrm{~J}_{\mathrm{PH}} 25.2 \mathrm{~Hz},{ }^{3} \mathrm{~J}_{\mathrm{HH}} 1.1 \mathrm{~Hz}, 1 \mathrm{H}, \mathrm{H}-\mathrm{C}-\mathrm{P}\right), 1.34\left(\mathrm{t},{ }^{3} \mathrm{~J}_{\mathrm{HH}} 7.1 \mathrm{~Hz}, 3 \mathrm{H}, \mathrm{CH}_{3^{-}}\right), 1.02-1.06(\mathrm{~m}$, $12 \mathrm{H},{ }^{\mathrm{t}} \mathrm{Bu}$ and $\left.\mathrm{CH}_{3}-\mathrm{CH}_{2}-\right), 0.26\left(\mathrm{~s}, 3 \mathrm{H},-\mathrm{Si}\left(\mathrm{CH}_{3}\right)_{2}\right), 0.25\left(\mathrm{~s}, 3 \mathrm{H},-\mathrm{Si}\left(\mathrm{CH}_{3}\right)_{2}\right) ; \delta_{\mathrm{C}}\left(176 \mathrm{MHz}, \mathrm{CDCl}_{3}\right) 163.1\left(\mathrm{~d},{ }^{2} \mathrm{~J}_{\mathrm{PC}} 5.2 \mathrm{~Hz}\right.$, O-C(O)), $143.6\left(C_{A r}\right), 143.1\left(C_{A r}\right), 136.8\left(C_{A r}\right), 125.3\left(C_{A r}\right), 125.0\left(C_{A r} H\right), 124.6\left(C_{A r}\right), 122.9\left(C_{A r} H\right), 122.1\left(C_{A r} H\right)$, $121.3\left(C_{A r} \mathrm{H}\right), 120.9\left(C_{A r} \mathrm{H}\right), 120.3\left(C_{A r} \mathrm{H}\right), 118.4\left(C_{A r} \mathrm{H}\right), 116.9\left(\mathrm{~d},{ }^{3} J_{\mathrm{PC}} 18.1 \mathrm{~Hz},-C_{A r}-\mathrm{C}_{A r} \mathrm{H}-\mathrm{N}\right), 111.7\left(C_{A r} \mathrm{H}\right), 63.4(\mathrm{~d}$, $\left.{ }^{2} J_{P C} 6.7 \mathrm{~Hz}, \mathrm{CH}_{3}-\mathrm{CH}_{2}-\mathrm{OP}\right), 63.2\left(\mathrm{~d},{ }^{2} J_{\mathrm{PC}} 6.7 \mathrm{~Hz}, \mathrm{CH}_{3}-\mathrm{CH}_{2}-\mathrm{OP}\right), 47.5$ (d, $\left.{ }^{1} J_{\mathrm{PC}} 123.9 \mathrm{~Hz},-\mathrm{CH}-\mathrm{P}\right), 34.8\left(\mathrm{~d},{ }^{3} J_{\mathrm{PC}} 3.2 \mathrm{~Hz},-\mathrm{C}_{\mathrm{Ar}}{ }^{-}\right.$ $\left.\mathrm{CH}-\mathrm{C}_{\mathrm{Ar}}\right), 25.8\left(\mathrm{C}-(\mathrm{CH} 3)_{3}\right), 25.8\left(\mathrm{C}-(\mathrm{CH} 3)_{3}\right), 16.4\left(\mathrm{~d},{ }^{2} \mathrm{~J}_{\mathrm{PC}} 6.2 \mathrm{~Hz}, \mathrm{CH}_{3}-\mathrm{CH}_{2}-\mathrm{OP}\right), 16.2\left(\mathrm{~d},{ }^{2} \mathrm{~J}_{\mathrm{PC}} 6.2 \mathrm{~Hz}, \mathrm{CH}_{3}-\mathrm{CH}_{2}-\mathrm{OP}\right),-4.3$ $\left(\mathrm{Si}-\mathrm{CH}_{2}\right),-4.4\left(\mathrm{Si}-\mathrm{CH}_{2}\right) ; \delta_{\mathrm{P}}\left(283.3 \mathrm{MHz}, \mathrm{CDCl}_{3}\right) 18.9 \mathrm{ppm}$.

8-((tert-Butyldimethylsilyl)oxy)-4-(1H-indol-3-yl)-3-methylenechroman-2-one (10). A mixture of diethyl $\left(\left(3 R^{*}, 4 S^{*}\right)-8-((\right.$ tert-butyldimethylsilyl)oxy)-4-(1H-indol-3-yl)-2-oxochroman-3-yl)phosphonate 9 (0.265 g, 0.5 $\mathrm{mmol})$ and $\mathrm{K}_{2} \mathrm{CO}_{3}(0.207 \mathrm{~g}, 1.5 \mathrm{mmol})$ in THF $(5 \mathrm{~mL})$ was stirred at $0{ }^{\circ} \mathrm{C}$ for $15 \mathrm{~min}$. Then aqueous formaldehyde $(40 \%, 0.20 \mathrm{~mL})$ was added and resulting suspension was stirred at $20{ }^{\circ} \mathrm{C}$ for an additional $3 \mathrm{~h}$. The mixture was then concentrated in vacuo and the solid residue was extracted with $\mathrm{CH}_{2} \mathrm{Cl}_{2}(3 \times 20 \mathrm{~mL})$. The combined organic layers were dried over $\mathrm{MgSO}_{4}$ and evaporated. The oily residue was subjected for column chromatography on silica gel using $\mathrm{CH}_{2} \mathrm{Cl}_{2} / \mathrm{Me}_{2} \mathrm{CO}(10: 1)$ as eluent $\left(R_{F} \sim 0.8\right)$ to afford methylenelactone 10 (0.122 $\left.\mathrm{g}, 60 \%\right)$. Colorless foam; Anal. calcd for $\mathrm{C}_{24} \mathrm{H}_{27} \mathrm{NO}_{3} \mathrm{Si}$ : C, 71.08; $\mathrm{H}, 6.71 ; \mathrm{N}, 3.45$; Found $\mathrm{C}, 71.3 ; \mathrm{H}, 6.7 ; \mathrm{N}, 3.5$; IR(ATR): $3406,2952,2929,2885,2857,1738,1478,1460,1295,1253,1187,1124,860,838,802,780,738 \mathrm{~cm}^{-1} ; \delta_{H}(700$ $\left.\mathrm{MHz}_{1} \mathrm{CDCl}_{3}\right) 8.10$ (bs, $\left.1 \mathrm{H}, \mathrm{NH}\right), 6.68-7.40\left(\mathrm{~m}, 8 \mathrm{H}, \mathrm{H}-\mathrm{C}_{\mathrm{Ar}}\right), 6.36\left(\mathrm{dd},{ }^{2} J_{\mathrm{HH}} 1.6 \mathrm{~Hz},{ }^{4} J_{\mathrm{HH}} 0.9 \mathrm{~Hz}, 1 \mathrm{H},=\mathrm{CHH}\right), 5.72(\mathrm{dd}$, $\left.{ }^{2} J_{\mathrm{HH}} 1.6 \mathrm{~Hz},{ }^{4} J_{\mathrm{HH}} 0.9 \mathrm{~Hz}, 1 \mathrm{H},=\mathrm{CHH}\right), 5.21$ (bs, $\left.1 \mathrm{H},-\mathrm{CH}-\mathrm{C}=\mathrm{CH}_{2}\right), 1.05\left(\mathrm{~s}, 12 \mathrm{H},{ }^{\mathrm{t}} \mathrm{Bu}\right.$ and $\left.\mathrm{CH}_{3}-\mathrm{CH}_{2}-\right), 0.27$ (s, $3 \mathrm{H},-$ $\left.\mathrm{Si}\left(\mathrm{CH}_{3}\right)_{2}\right), 0.26\left(\mathrm{~s}, 3 \mathrm{H},-\mathrm{Si}\left(\mathrm{CH}_{3}\right)_{2}\right) ; \delta_{\mathrm{C}}\left(176 \mathrm{MHz}, \mathrm{CDCl}_{3}\right) 163.3(\mathrm{O}-\mathrm{C}(\mathrm{O})), 143.9\left(C_{A r}\right), 142.3\left(C_{A r}\right), 137.0\left(C_{A r}\right), 136.4$ $(=C<), 127.9\left(=\mathrm{CH}_{2}\right), 126.8\left(C_{A r}\right), 125.6\left(C_{A r}\right), 124.7\left(C_{A r} \mathrm{H}\right), 123.4\left(C_{A r} \mathrm{H}\right), 122.6\left(C_{A r} \mathrm{H}\right), 120.8\left(C_{A r} \mathrm{H}\right), 120.6\left(C_{A r} \mathrm{H}\right)$, $119.9\left(C_{A r} \mathrm{H}\right), 119.7\left(C_{A r} \mathrm{H}\right), 114.4\left(C_{A r}\right), 111.7\left(C_{A r} \mathrm{H}\right), 40.75\left(-\mathrm{C}_{A r}-C H-C_{A r}\right), 25.9\left(-\mathrm{C}-\left(C_{3}\right)_{3}\right), 18.6\left(-C-\left(\mathrm{CH}_{3}\right)_{3}\right),-4.4(-$ $\left.\mathrm{Si}\left(\mathrm{CH}_{3}\right)_{2}\right)$.

8-Hydroxy-4-(1H-indol-3-yl)-3-methyl-2H-chromen-2-one (11). To a stirred solution of compound 10 (0.101 g, $0.25 \mathrm{mmol})$ in anhydrous THF $(2 \mathrm{~mL})$ was added a solution of TBAF in THF (1.1M, $0.45 \mathrm{~mL}, 0.50 \mathrm{mmol})$. The mixture was stirred at rt overnight until disappearance of the starting material (TLC). After evaporation in vacuo the residue was purified by flash chromatography $\left(\mathrm{CH}_{2} \mathrm{Cl}_{2} / \mathrm{Me}_{2} \mathrm{CO} 10: 1\right)$ to yield 11 (0.052 $\left.\mathrm{g}, 71 \%\right)$. Colorless foam; Anal. calcd for $\mathrm{C}_{18} \mathrm{H}_{13} \mathrm{NO}_{3}$ : C, 74.22; $\mathrm{H}, 4.50 ; \mathrm{N}, 4.81$; Found C, 74.4; $\mathrm{H}, 4.5 ; \mathrm{N}, 4.8 ; \mathrm{IR}(\mathrm{ATR})$ : $3363,3187,2983,1668,1570,1420,1384,1369,1310,1139,1092,1036,751,735,512 \mathrm{~cm}^{-1} ; \delta_{H}(700 \mathrm{MHz}$, acetone-d6) 10.80 (bs, $1 \mathrm{H}, \mathrm{NH}), 8.81$ (bs, $1 \mathrm{H}, \mathrm{OH}), 7.58$ (bd, $\left.{ }^{3} \mathrm{~J}_{\mathrm{HH}} 8.2 \mathrm{~Hz} 1 \mathrm{H}, \mathrm{C}_{\mathrm{Ar}} \mathrm{H}-\mathrm{C}_{\text {Ind }}-\mathrm{C}_{\text {Ind }}\right), 7.55\left(\mathrm{~s}, 1 \mathrm{H}, \mathrm{C}_{\mathrm{Ar}} \mathrm{H}-\mathrm{NH}\right)$, $7.29\left(\mathrm{bd},{ }^{3} J_{\mathrm{HH}} 8.0 \mathrm{~Hz}, 1 \mathrm{H}, \mathrm{C}_{\mathrm{Ar}} \mathrm{H}-\mathrm{C}_{\text {Ind }}-\mathrm{NH}\right), 7.22\left(\mathrm{dt},{ }^{3} J_{\mathrm{HH}} 7.6 \mathrm{~Hz},{ }^{4} J_{\mathrm{HH}} 0.8 \mathrm{~Hz}, 1 \mathrm{H}, \mathrm{C}_{\text {Ind }} \mathrm{H}-\mathrm{C}_{\text {Ind }} \mathrm{H}-\mathrm{C}_{\text {Ind }}-\mathrm{C}_{\text {Ind }}\right), 7.06-7.09$ (m, $\left.2 \mathrm{H}, H_{\mathrm{Ar}}\right), 7.00\left(\mathrm{t},{ }^{3} J_{\mathrm{HH}} 7.7 \mathrm{~Hz}, 1 \mathrm{H}, \mathrm{C}_{\mathrm{Ar}} \mathrm{H}-\mathrm{C}_{\mathrm{Ar}} \mathrm{H}-\mathrm{C}_{\mathrm{Ar}} \mathrm{H}\right), 6.81\left(\mathrm{dt},{ }^{3} J_{\mathrm{HH}} 8.0 \mathrm{~Hz},{ }^{4} J_{\mathrm{HH}} 1.3 \mathrm{~Hz}, 1 \mathrm{H}, H_{\mathrm{Ar}}\right), 2.02\left(\mathrm{~s}, 3 \mathrm{H},-\mathrm{CH}_{3}\right) ; \delta_{\mathrm{C}}$ $\left(176 \mathrm{MHz}, \mathrm{CDCl}_{3}+10 \% \mathrm{CD}_{3} \mathrm{OD}\right) 163.2(\mathrm{O}-\mathrm{C}(\mathrm{O})), 146.8\left(C_{\mathrm{Ar}}\right), 144.1\left(C_{\mathrm{Ar}}\right), 140.8\left(C_{\mathrm{Ar}}\right), 136.1\left(C_{\mathrm{Ar}}\right), 126.4\left(C_{\mathrm{Ar}}\right)$, 
$124.8\left(C_{\mathrm{Ar}} \mathrm{H}\right), 124.0\left(C_{\mathrm{Ar}} \mathrm{H}\right), 123.0\left(C_{\mathrm{Ar}}\right), 122.4\left(C_{\mathrm{Ar}} \mathrm{H}\right), 121.8\left(C_{\mathrm{Ar}}\right), 120.1\left(C_{\mathrm{Ar}} \mathrm{H}\right), 119.7\left(C_{\mathrm{Ar}} \mathrm{H}\right), 118.4\left(C_{\mathrm{Ar}} \mathrm{H}\right), 117.4$ $\left(C_{\mathrm{Ar}} \mathrm{H}\right), 111.7\left(C_{\mathrm{Ar}} \mathrm{H}\right), 109.6\left(C_{\mathrm{Ar}}\right), 15.1\left(-\mathrm{CH}_{3}\right)$.

\section{Supplementary Material}

Supplementary material containing copies of $I R,{ }^{1} \mathrm{H}$ and ${ }^{13} \mathrm{C}$ NMR spectra associated with this paper can be found in the online version.

\section{References}

1. Horton, D. A.; Bourne, G. T.; Smythe, M. L. Chem. Rev. 2003, 103, 893-930. http://dx.doi.org/10.1021/cr020033s

2. Welsch, M.E.; Snyder, S.A.; Stockwell, B.R. Curr. Opin. Chem. Biol. 2010, 14, 347-361. http://dx.doi.org/10.1016/i.cbpa.2010.02.018

3. Grover. J; Jachak, S.M. RSC Adv. 2015, 5, 38892-38905. http://dx.doi.org/10.1039/c3ob42582g

4. Zhan, Z.-P.; Yang, R.-F.; Lang, K. Tetrahedron Lett. 2005, 46, 3859-3862. http://dx.doi.org/10.1016/j.tetlet.2005.03.174

5. Bartoli, G.; Bosco, M.; Giuli, S.; Giuliani, A.; Lucarelli, L.; Marcantoni, E.; Sambri, L.; Torregiani, E. J. Org. Chem. 2005, 70, 1941-1944. http://dx.doi.org/10.1021/jo048776w

6. Harrington, P. E.; Kerr, M. A. Synlett 1996, 1047-1048. http://dx.doi.org/10.1055/s-1996-568

7. Yadav, J. S.; Abraham, S.; Reddy, B. V. S.; Sabitha, G. Synthesis 2001, 2165-2169. http://dx.doi.org/10.1055/s-2001-18068

8. Bandini, M.; Melchiorre, P.; Melloni, A.; Umani-Ronchi, A. Synthesis 2002, 1110-1114. http://dx.doi.org/10.1055/s-2002-31970

9. Lin, C. C.; Hsu, J. M.; Sastry, M. N. V.; Fang, H. L.; Tu, Z. J.; Liu, J. T.; Yao, C.F. Tetrahedron 2005, 61, 1175111757. http://dx.doi.org/10.1016/i.tet.2005.09.038

10. Li-Tao, A.; Jian-Ping, Z.; Li-Li, Z.; Yong, Z. Tetrahedron Lett. 2007, 48, 4297-4300. http://dx.doi.org/10.1016/j.tetlet.2007.04.011

11. Meshram, H. M.; Nageswara Rao, G.; Santosh Kumar, G. Synth. Commun. 2010, 40, 3496-3500. http://dx.doi.org/10.1080/00397910903457316

12. Alam, M. M.; Varala, R.; Adapa, S. R. Tetrahedron Lett. 2003, 44, 5115-5119. http://dx.doi.org/10.1016/S0040-4039(03)01089-X

13. Azizi, N.; Arynasab, F.; Saidi, M. R. Org. Biomol. Chem. 2006, 4, 4275-4277. http://dx.doi.org/10.1039/B610263H

14. Ballini, R.; Clemente, R. R.; Palmieri, A.; Petrini, M. Adv. Synth. Catal. 2006, 348, 191-196. http://dx.doi.org/10.1002/adsc.200505339

15. Wu, P.; Wan, Y.; Cai, J. Synlett 2008, 1193-1198. http://dx.doi.org/10.1055/s-2008-1072735 
16. Li-Tao, A.; Li-Li, Z.; Jian-Ping, Z.; Guang-Liang, Z. Synth. Commun. 2010, 40, 1978-1984. http://dx.doi.org/10.1080/00397910903219344

17. Kumar, V. P.; Sridhar, R.; Srinivas, B.; Narender, M.; Rao, K. R. Can. J. Chem. 2008, 86, 907-911. http://dx.doi.org/10.1139/v08-118

18. Sri Hari, G.; Nagaraju, M.; Marthanda Murthy, M. Synth. Commun. 2008, 38, 100-105. http://dx.doi.org/10.1080/00397910701650815

19. Xie, J.; Zhu, X.; Huang, M.; Meng, F.; Wang, M.; Wan, Y. Synth. Commun. 2010, 40, 3259-3267. http://dx.doi.org/10.1080/00397910903398676

20. Jeganathan, M.; Kanagaray, K.; Dhakshinamoorthy, A.; Pitchumani, K. Tetrahedron Lett. 2014, 55, 20612064.

http://dx.doi.org/10.1016/j.tetlet.2014.01.112

21. Liang, D.; Li, X.; Zhang, W.; Li, Y.; Zhang, M.; Cheng, P. Tetrahedron Lett. 2016, 57, 1027-1030. http://dx.doi.org/10.1016/j.tetlet.2016.01.078

22. Mane, A.; Lohar, T.; Salunkhe, R. Tetrahedron Lett. 2016, 57, 2341-2346. http://dx.doi.org/10.1016/j.tetlet.2016.04.057

23. Shiri, M. Chem. Rev. 2012, 112, 3508-3549. http://dx.doi.org/10.1021/cr2003954

24. Oikawa, Y.; Hirasawa, H.; Yonemitsu, O. Tetrahedron Lett. 1978, 19, 1759-1762. http://dx.doi.org/10.1016/0040-4039(78)80037-9

25. Oikawa, Y.; Hirasawa, H.; Yonemitsu, O. Chem. Pharm. Bull. 1982, 30, 3092-3096. http://dx.doi.org/10.1248/cpb.30.3092

26. Boisbrun, M.; Kovács-Kulyassa, A.; Jeannin, L.; Sapi, J.; Toupet, L.; Laronze J-Y. Tetrahedron Lett. 2000, 41, 9771-9775. http://dx.doi.org/10.1016/S0040-4039(00)01719-6

27. Nickerson, D.M.; Mattson, A.E. Chem. Eur. J. 2012, 18, 8310-8314. http://dx.doi.org/10.1002/chem.201201206

28. Oelerich, J.; Roelfes, G. Org. Biomol. Chem. 2015, 13, 2793-2799. http://dx.doi.org/10.1039/c4ob02487g

29. Zhuang, W.; Hansen, T.; Jørgensen, K. A. Chem. Commun. 2001, 347-348. http://dx.doi.org/10.1039/B009008P

30. Zhou, J.; Tang, Y. J. Am. Chem. Soc. 2002, 124, 9030-9031. http://dx.doi.org/10.1021/ja026936k

31. Zhou, J.; Ye, M.-C.; Huang, Z.-Z.; Tang, Y. J. Org. Chem. 2004, 69, 1309-1320. http://dx.doi.org/10.1021/jo035552p

32. Zhou, J.; Tang, Y. Chem. Commun. 2004, 432-433. http://dx.doi.org/10.1039/B313197A

33. Sun, Y.-J.; Li, N.; Zheng, Z.-B.; Liu, L.; Yu, Y.-B.; Qin, Z.-H.; Fu, B. Adv. Synth. Catal. 2009, 351, 3113-3117. http://dx.doi.org/10.1002/adsc.200900669

34. Wen, L.; Shen, Q.; Wan, X.; Lu, L. J. Org. Chem. 2011, 76, 2282-2285. http://dx.doi.org/10.1021/jo1024333

35. Liu, Y.; Zhou, X.; Shang, D.; Liu, X.; Feng, X. Tetrahedron 2010, 66, 1447-1457. http://dx.doi.org/10.1016/i.tet.2009.12.032

36. Ye, M.-C.; Yang, Y.-Y.; Tang, Y.; Sun, X.-L.; Ma, Z.; Qin, W.-M. Synlett 2006, 8, 1240-1244. http://dx.doi.org/10.1055/s-2006-932472 
37. Kumar, A.; Kumar, P.; Tripathi, V. D.; Srivastava, S. RSC Adv. 2012, 2, 11641-11644. http://dx.doi.org/10.1039/c2ra21284f

38. Shao, Z.; Xu, L.; Wang, L.; Wei, H.; Xiao, J. Org. Biomol. Chem. 2014, 12, 21852188. http://dx.doi.org/10.1039/c3ob42582g

39. Deredas, D.; Huben, K.; Maniukiewicz, W.; Krawczyk, H. Tetrahedron 2014, 70, 8925-8929. http://dx.doi.org/10.1016/j.tet.2014.09.064

40. Deredas, D.; Huben, K.; Maniukiewicz, W.; Krawczyk, H. Synlett 2014, 25, 280-282. http://dx.doi.org/10.1055/s-0033-1340347

41. Krawczyk, H.; Albrecht, Ł.; Wojciechowski, J.; Wolf, W. M.; Krajewska, U.; Różalski, M. Tetrahedron 2008, 64, 6307-6314. http://dx.doi.org/10.1016/i.tet.2008.04.090

42. Deredas, D.; Albrecht, Ł.; Krawczyk, H. Tetrahedron Lett. 2013, 54, 3088-3090. http://dx.doi.org/10.1016/i.tetlet.2013.03.130

43. Bojilova, A.; Nikolova, R.; Ivanov, C.; Rodios. N. A.; Terzis, A.; Raptopolou, C. P. Tetrahedron 1996, 52, 12597-12612. http://dx.doi.org/10.1016/0040-4020(96)00748-X

44. Guo, T.; Liu, Y.; Zhao, Y-H.; Zhang, P-K.; Han, S-L.; Liu, H-M. Tetrahedron Lett. 2016, 57, 4629-4632. http://dx.doi.org/10.1016/j.tetlet.2016.09.012 